\title{
Investigation of the Mechanisms of Antimicrobial Peptides Interacting with Membranes by Fluorescence Correlation Spectroscopy
}

\author{
Lanlan $\mathrm{Yu}^{1,2}$, Jeak Ling Ding ${ }^{3}$, Bow $\mathrm{Ho}^{4}$, Si-Shen Feng ${ }^{5}$ and Thorsten Wohland ${ }^{*}, 1$ \\ ${ }^{I}$ Department of Chemistry, National University of Singapore, 3 Science Drive 3, Singapore 117543 \\ ${ }^{2}$ Department of Chemistry, Zhengzhou University, Zhengzhou 450001, P.R. China \\ ${ }^{3}$ Department of Biological Sciences, National University of Singapore, 14 Science Drive 4, Singapore 117543 \\ ${ }^{4}$ Department of Microbiology, National University of Singapore, 5 Science Drive 2, Singapore 117597 \\ ${ }^{5}$ Division of Bioengineering, Chemical \& Biomolecular Engineering, National University of Singapore, 10 Kent Ridge \\ Crescents, Singapore 119260
}

\begin{abstract}
The interaction of four antimicrobial peptides, including magainin 2, melittin, polymyxin B and an artificial peptide V4, with phospholipid membranes was investigated by fluorescence correlation spectroscopy (FCS) and confocal imaging. Fluorophore entrapping and labeled vesicles were used to quantitatively determine the extent of antimicrobial peptides interacting with membrane mimics and unravel their different mechanisms of action. It is shown that at high peptide/lipid ratio, magainin 2 and melittin form pores to induce comparable level of membrane permeation while polymyxin $\mathrm{B}$ and V4 disrupt the membrane. Considering its low solubility, V4 is much more active than others. Due to the hydrophobic interactions, melittin, polymyxin B and V4 all promote aggregation when they perform their function. These experiments demonstrate the feasibility of mechanistic studies by an FCS based approach.
\end{abstract}

Keywords: Antimicrobial peptides, magainin 2, melittin, polymyxin B, V4, fluorescence correlation spectroscopy, peptidelipid interaction.

\section{INTRODUCTION}

Antimicrobial peptides are important defense weapons of many organisms. They target bacterial membranes, inhibit the growth of bacteria and finally lead to their death [1-3]. Because of the difficulty for bacteria to develop a new membrane system, antimicrobial peptides that mainly target the bacterial membrane have been considered promising drug candidates as a substitute for conventional antibiotics for which many pathogens have acquired a variety of resistance. Therefore, in an effort to defend against bacterial infections, the investigation of the functional mechanism of antimicrobial peptides against bacterial membrane lipids has become an important subject.

Antimicrobial peptides which represent the elements of innate immunity are widely found in a variety of host organisms including insects, amphibians and mammals $[4,5]$. Based on their secondary structures, most antimicrobial peptides are divided into $\alpha$-helix, $\beta$-sheet, peptides with cyclic loop structures and peptides rich in certain amino acids $[6$, 7]. The representative antimicrobial peptides include magainins, cecropins, defensins, indolicidin and melittin [8-10]. Magainin 2 which was first discovered in the skin of the African clawed frog Xenopus has been extensively investigated

*Address correspondence to this author at the Department of Chemistry, National University of Singapore, 3 Science Drive 3, Singapore 117543;

E-mail: chmwt@nus.edu.sg
[9]. This peptide consists of 23 amino acids and bears a positive charge of +4 at physiological conditions. When magainin 2 interacts with a lipid membrane, an $\alpha$-helical conformation is induced with the interface parallel to the membrane surface [11]. Magainin 2 is an efficient antimicrobial peptide with broad spectrum antimicrobial activity, killing gram-negative bacteria, gram-positive bacteria, fungi and protozoa as well as targeting some tumors. The minimum inhibitory concentration for mammalian cells is 10-100 times higher than that for various microorganisms [12], which indicates that magainin 2 has high potential to be a new drug candidate. Melittin is a toxic antimicrobial peptide from bee venom which kills both bacteria and mammalian cells. It consists of 26 amino acids and bears a positive charge of +5 to +6 [13-15]. Similar to magainin 2, melittin adopts an $\alpha$ helical conformation upon interaction with membranes [14]. Polymyxin B which was isolated from Bacillus polymyxa is an important cyclic cationic antimicrobial peptide [16-18]. Similar to melittin, despite the antimicrobial activity, this peptide is also highly toxic which limits its usage in the prophylaxis and therapy of sepsis [16]. The interaction of polymyxin B with lipopolysaccharides (LPS), which is the major component of the outer membrane of gram-negative bacteria related to the majority of cases of sepsis, or lipid A, which is the bioactive part of LPS, has been widely studied $[17,19,20]$. However, information is lacking on the interaction of polymyxin B with the components of cytoplasmic membranes, including negatively charged phospholipids. 
Besides the naturally occurring antimicrobial peptides, many designed or modified antimicrobial peptides also show strong antimicrobial activity and reduced toxicity indicating a high potential for drug development [21,22]. Our previously de novo designed antimicrobial peptide $\mathrm{V} 4$ which is a cyclic peptide, shows a good combination of high antimicrobial activity, low cytotoxic and low hemolytic activity compared to the control peptide polymyxin B [23]. Besides the high binding activity for LPS and lipid A, V4 also displays a higher affinity for negatively charged phospholipids than for zwitterionic phospholipids [24]. The strong interaction of V4 to the negatively charged lipids showed that electrostatic force is a prerequisite for its selective action on bacterial membranes, in contrast to mammalian membranes.

It is believed that many antimicrobial peptides perform their function by a process named self-promoted uptake $[6$, $25,26]$. Cationic antimicrobial peptides first distort the outer membrane of the bacteria, leading to the passage of the antimicrobial peptides through the outer membrane and interaction with the cytoplasmic membranes. The antimicrobial peptides then form pores or disrupt the cytoplasmic membrane and eventually lead to bacterial death. Currently, two main mechanisms have been proposed to describe this process, the barrel-stave and the carpet model [7]. The barrelstave model envisions the formation of transmembrane pores with $\alpha$-helices forming bundles. However the carpet mechanism proposes that antimicrobial peptides initially bind to the membrane surface followed by the disruption of the membrane in a detergent-like manner. Some researchers argued that before membrane disruption, transient holes in the membrane, named toroidal (wormhole) pores, could be formed [7]. Magainin 2 and melittin are believed to induce membrane permeation by forming toroidal pores. However, the structure of the pore is still controversial $[27,28]$. Because the component of the bacterial cytoplasmic membrane which is rich in negatively charged phospholipids is different from that of mammalian cell membranes, two negatively charged phospholipids (1-Palmitoyl-2-Oleoyl-sn-Glycero-3[Phospho-rac-(1-glycerol)] (POPG) and 1,2-Dipalmitoyl-snGlycero-3-[Phospho-rac-(1-glycerol)] (DPPG)) were chosen in the present study as bacterial membrane models to investigate the mechanism of antimicrobial peptides such as magainin 2, melittin, polymyxin B and V4 to induce membrane permeation. POPG and DPPG are representative unsaturated and saturated phospholipids, respectively, which might be used for investigation of the effect of lipid saturation on peptide activity. Fig. (1) shows two membrane models, fluorophore-entrapping vesicles and fluorophore-labeled vesicles [29], which were used in this study to identify pore formation and membrane disruption. Detergent Triton-X100 was used for complete leakage control.

In the present work, we used fluorescence correlation spectroscopy (FCS) to investigate the mechanisms of antimicrobial peptides interacting with membranes. Conventional fluorescent measurement is widely used for interactions [30]. However, FCS, as a sensitive technique, has been used successfully to study the interaction of antimicrobial peptides with membranes [29, 31, 32]. Especially the application of fluorophore-entrapping vesicles and fluorophore-labeled vesicles provides a new method for detection of the vesicle leak-
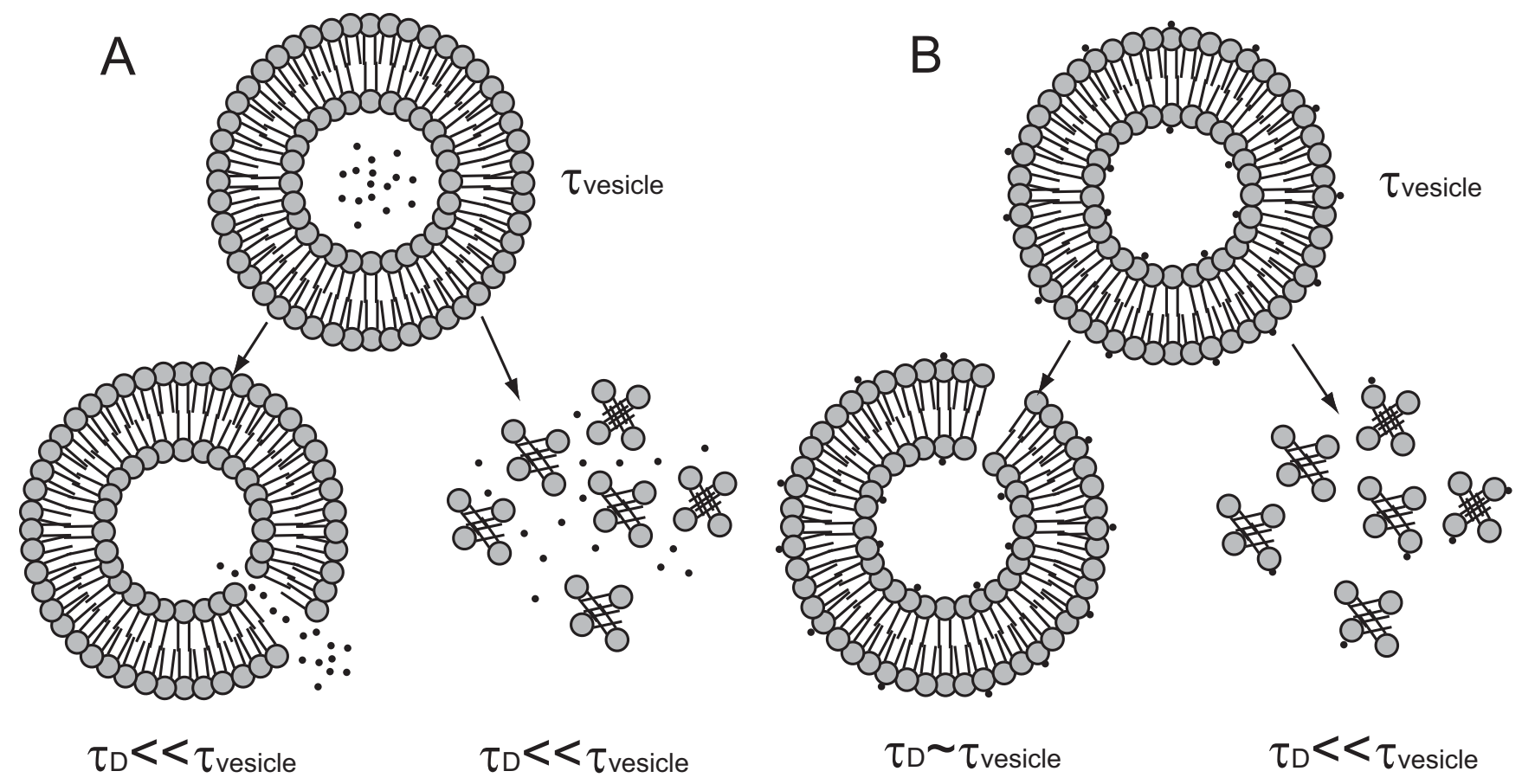

Fig. (1). Schematic drawing of the investigation of the mechanisms of antimicrobial peptides by REVs and RLVs. (A) REVs with the diffu-

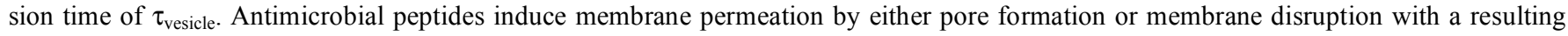

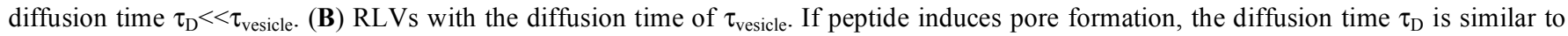

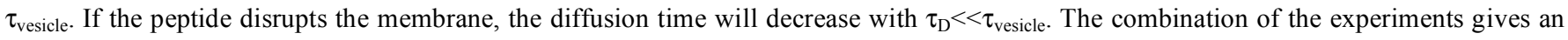
answer to whether pore formation or membrane disruption is the mechanism of action. 
age [29]. However, studies up to the present only provided qualitative information about the action of antimicrobial peptides on vesicle-pore formation or disruption, showing the antimicrobial peptides' activity at a particular peptide/lipid ratio. In this study, we consider the interaction between antimicrobial peptides and vesicles over a wide peptide/lipid ratio range. Moreover, we provide quantitative calculation of the number of vesicle and fraction of vesicle in the mixture, showing the gradual effect of peptide concentration on the process of membrane leakage.

In this work, we used FCS to (i) quantitatively investigate the interaction between the antimicrobial peptides: magainin 2, melittin, polymyxin B and V4 and POPG and DPPG membranes, (ii) compare the ability of these four antimicrobial peptides to induce membrane permeation and (iii) study the mechanisms of interaction between the above named antimicrobial peptides with these membrane models. To support the FCS results, we have used confocal microscopy to show the action of the different peptides on lipid vesicles.

\section{MATERIALS AND METHODS}

\section{Materials}

Rhodamine 6G (Rho 6G) is a product from Molecular Probes (ITS Science and Medical Pte Ltd, Singapore). POPG, DPPG, 1-Palmitoyl-2- Oleoyl-sn-Glycero-3Phosphocholine (POPC) and 1,2-Dipalmitoyl-sn-Glycero-3Phospho-ethanolamine-N-(Lissamine Rhodamine B Sulfonyl) (Ammonium Salt) (Rho-PE) were purchased from Avanti (Avanti Polar Lipids, Inc., Alabaster, AL). Antimicrobial peptides magainin 2 (M2), melittin (ME), polymyxin B (PB), Triton X-100 and phosphate buffered saline (PBS) were purchased from Sigma-Aldrich (Sigma-Aldrich Pte Ltd, Singapore). The purity of magainin 2 and melittin is $99 \%$ and $93 \%$, respectively. Polymyxin B is a mixture of polymyxin $\mathrm{B} 1$ and polymyxin $\mathrm{B} 2$. V4 peptide (CVKVQV KVGSGVKVQVKVC) with cyclization by a disulfide bond at the two terminal cysteines (C) was synthesized by Genemed (Genemed Synthesis, Inc., South San Francisco, CA). The purity of V4 was above $97 \%$. All the materials were used without further purification.

\section{Rhodamine 6G Entrapping LUVs (REVs)}

POPG and DPPG were prepared as stock solutions in chloroform and a mixture of chloroform and ethanol $(\mathrm{v} / \mathrm{v}$ 4:1) respectively. The solvent was evaporated under $\mathrm{N}_{2}$ gas and then the sample was placed into vacuum for at least one hour. PBS which includes $1 \mu \mathrm{M}$ Rho $6 \mathrm{G}$ was added to redissolve the lipids to yield an aqueous suspension of phospholipids at a lipid concentration of $0.5 \mathrm{mM}$. LUVs were prepared by freeze-thawing the lipid suspension 5 times followed by extrusion through $0.1 \mu \mathrm{m}$ polycarbonate membrane filters for 20 times using a mini-extruder syringe device (Avanti Polar Lipids). For DPPG lipid, because of the high transition temperature, heat was needed to increase the temperature above the transition temperature during the extrusion process. After extrusion, MicroSpin ${ }^{\mathrm{TM}}$ S-200 HR Columns (Amersham Biosciences, Singapore) were used to remove non-entrapped Rho $6 \mathrm{G}$ from the vesicle solution [29].

\section{Rho-PE Labeled LUVs (RLVs)}

The preparation of labeled LUVs followed a similar protocol as above. Phospholipid was mixed with a small percentage of Rho-PE in a volatile organic solvent. After completely removing the solvent, PBS was added to form a suspension of phospholipids. RLVs were obtained by freezethawing the lipid suspension 5 times followed by extrusion for 20 times through $0.1 \mu \mathrm{m}$ polycarbonate membrane filters. For FCS measurements, RLVs were prepared with a content of $0.1 \%$ Rho-PE. Confocal pictures were taken with LUVs that contained 1\% Rho-PE. Because the percentage of RhoPE was very low, RLVs can be used to represent the lipid vesicles under investigation.

\section{Fluorescence Correlation Spectroscopy (FCS)}

FCS analyzes fluorescence intensity (photon count rate) fluctuations caused by minute deviations from thermal equilibrium from a small confocal volume in a sample which contains fluorescent particles. Its theory and application can be found in many reviews [33-42]. For 3D diffusion processes, the measured fluorescence photon count rate autocorrelation function (ACF) $G(\tau)$ can be fitted as follows:

$$
G(\tau)=\frac{1}{N} \frac{\sum_{i=1}^{n}\left(\frac{Q_{i}}{Q_{1}}\right)^{2} F_{i} g_{i}(\tau)}{\left[\sum_{i=1}^{n} \frac{Q_{i}}{Q_{1}} F_{i}\right]^{2}}+G_{\infty}
$$

with

$g_{i}(\tau)=\left(1+\frac{\tau}{\tau_{D i}}\right)^{-1}\left(1+\frac{\tau}{\left(\frac{z}{\omega}\right)^{2} \tau_{D i}}\right)^{-1 / 2}$

and the diffusion time of species $i$

$$
\tau_{D i}=\frac{\omega^{2}}{4 D_{i}}
$$

$N$ is the average number of particles in the confocal volume [43-46]. To recover absolute concentrations, we calibrated the system with a $1 \mathrm{nM}$ fluorophore (Rho 6G) solution and set all other values in relation to this calibration. The fluorescence yields, $Q_{i}$, are the product of the extinction coefficient, the quantum yield, and the overall detection efficiency for particle $i$ in the instrument. The coefficients $F_{i}$ are the mole fraction of species $i$ in the sample and $g_{i}(\tau)$ is the characteristic function of the underlying process that causes the fluctuations. $\tau_{\mathrm{Di}}$ is the lateral diffusion time of the fluorescent particle staying in the confocal volume. $D_{i}$ is the diffusion coefficient of species $i$, and $\omega$ and $\mathrm{z}$ are the radial and axial distances of the confocal volume at which the intensity has dropped by $1 / \mathrm{e}^{2}$ of the maximum value. $G_{\infty}$ is the convergence value of the $\mathrm{ACF}$ for long times, in general this value is 1. The program IgorPro (Wavemetrics, Lake Oswego, OR, USA) was used to fit the autocorrelation function to the experimental data as described previously [47]. 
In solutions with two different fluorophores, the autocorrelation amplitude which is inversely proportional to the apparent number of particles $N_{a p p}$ is given by (Eq. 1 with $i=2$ ):

$$
G(0)-G_{\infty}=\frac{1}{N_{a p p}}=\frac{1}{N} \times \frac{F_{1}+\left(\frac{Q_{2}}{Q_{1}}\right)^{2} F_{2}}{\left(F_{1}+\left(\frac{Q_{2}}{Q_{1}}\right) F_{2}\right)^{2}}=\frac{N_{1}+\left(\frac{Q_{2}}{Q_{1}}\right)^{2} N_{2}}{\left(N_{1}+\left(\frac{Q_{2}}{Q_{1}}\right)_{2}\right)^{2}}
$$

with

$$
N=N_{1}+N_{2}
$$

where $N_{l}$ and $N_{2}$ are the particle number of species 1 and 2, respectively $[48,49]$.

\section{FCS Instrumentation}

FCS experiments were performed using an Axiovert 200 inverted microscope (Carl Zeiss South East Asia, Singapore). The laser was focused on the samples using a water immersion objective (C-Apochromat, 63×, NA 1.2, Zeiss). A dichroic filter (Omega Optical Inc., Brattleboro, VT) and an emitter (Omega) were used to separate the excitation light from the emission fluorescence. The samples were excited with the $515 \mathrm{~nm}$ line of laser beam from an Argon-Krypton laser (Melles Griot SP, Pte Ltd, Singapore). A $50 \mu \mathrm{m}$ diameter pinhole in the image plane blocked signals which were out-of-focus. The emitted fluorescence was detected by an avalanche photo diode detector (APD) (PerkinElmer Canada Inc., Canada) and then the signals were sent to a digital correlator (Model: Flex02-12D, www.correlator.com, Bridgewater, NJ) to be autocorrelated. The longest measurement time was 150 seconds. A cover was put on the coverslip and constant air humidity was kept during the experiment to avoid water evaporation. The error bars were based on the standard deviation from 5 complete sets of experiments.

\section{Interaction of Antimicrobial Peptides with REVs}

The stock solution of REVs was diluted and mixed with different concentrations of magainin 2, melittin, polymyxin B, V4 and detergent Triton X-100 individually. FCS experiments were performed after one hour incubation at room temperature. The final lipid concentration was $40 \mu \mathrm{M}$. The concentrations of antimicrobial peptides were 1, 2, 5, 10, 15 and $20 \mu \mathrm{M}$ with corresponding peptide/lipid ratio $(\mathrm{P} / \mathrm{L})$ of $1: 40,1: 20,1: 8,1: 4,1: 2.67$ and $1: 2$. The laser power was 100 $\mu \mathrm{W}$. A dichroic filter (525DRLP) and an emitter (545AF35) were used in experiments.

\section{Interaction of Antimicrobial Peptides with RLVs}

The same protocol was applied on the interaction of antimicrobial peptides with RLVs. The final lipid concentration was $40 \mu \mathrm{M}$. The $\mathrm{P} / \mathrm{L}$ were 1:40, 1:20, 1:8, 1:4, 1:2.67 and 1:2. The laser power was set at $40 \mu \mathrm{W}$, with the matching dichroic filter (560DRLP) and emitter (595AF60).

\section{Fluorescence Confocal Imaging}

$1 \%$ RLVs were diluted and mixed with antimicrobial peptides with different concentrations. The final lipid concentration was kept at $40 \mu \mathrm{M}$. The vesicle and peptide mix- ture was incubated for $5 \mathrm{~min}$ and placed on the coverslip (0.17 mm thick, Fisher Scientific Pte Ltd, Singapore) for measurements. Confocal imaging was performed on a FluoViewTM FV300 system (Olympus Singapore Pte Ltd, Singapore) with a HeNe laser $(543 \mathrm{~nm})$. A water-immersion objective (UPlanApo, 60X, NA1.2, Olympus) was used, in addition to a long pass filter at $560 \mathrm{~nm}$. A stack of 25 confocal images above the coverslip surface was acquired from bottom to top with a step size of $1 \mu \mathrm{m}$. The 25 confocal images were overlapped for results.

\section{RESULTS}

\section{Calibration}

The parameters used in the evaluation of the ACF to investigate the interaction of antimicrobial peptides with phospholipid membranes are a) the amplitude and thus the number of particles $N$ in the observation volume, b) the diffusion time $\tau_{\mathrm{D}}$, giving access to the diffusion coefficient of the particles, and c) the counts per particle and second, cps, i.e. the molecular brightness. All values for the calibrations are given in Table 1. Briefly, Rho 6G had a diffusion time of $\tau_{\mathrm{D}}$ $=51.5 \pm 0.8 \mu \mathrm{s}$ and a structure factor $\mathrm{z} / \omega=5$, which was kept constant in all other evaluations (Eq. 2). The cps of Rho $6 \mathrm{G}$, obtained by the average photon count rates divided by the particle number, was $60 \mathrm{kHz}$. POPG and DPPG RLVs were sufficiently well fitted with a single particle model representing the labeled vesicles with diffusion times between 4.4 - $4.8 \mathrm{~ms}$. For POPG and DPPG REVs a two particle model was necessary to fit the data adequately. In these cases the first fast diffusion time had a value around $44 \mu$ s representing free dye in solution which was not completely removed by column filtration, and the second slower diffusion time was between $2.8-4.4 \mathrm{~ms}$, representing the REVs. According to equation 4 , in a two species system, if the fluorescence yields of the two species are the same, the amplitude of the ACF is inversely proportional to the particle number, $N$. However, if the fluorescence yields of the two species are dissimilar, the fluorescence yield of the particles will affect the amplitude of the ACF and thus the real particle number $N$ in the confocal volume due to the different contribution of the two fluorescent species to the ACF. In this case the fluorescence yield of Rho $6 \mathrm{G}\left(Q_{1}\right)$ was $60 \mathrm{kHz}$. The particle number of the vesicles $N_{\text {vesicle }}\left(N_{2}\right)$ can be obtained from the RLVs at the same concentration. The efficiency of the column filtration was examined by the RLVs, showing that there was no substantial loss of vesicles (data not shown). Thus the vesicle number $N_{\text {vesicle }}\left(N_{2}\right)$ of REVs is assumed to be the same as that of RLVs under the same condition. Therefore, by fitting the experimental data with a twoparticle model with varying fluorescence yield of REVs, $Q_{2}$, the particle number of Rho $6 \mathrm{G}, N_{\text {rho }}\left(N_{l}\right)$, and thus the total particle number $N$ are obtained. The fluorescence yield of the POPG REVs was deduced to be $630 \mathrm{kHz}$ which was 10.5 times as bright as Rho $6 \mathrm{G}$. The particle number of Rho $6 \mathrm{G}$ and POPG REVs in the confocal volume was calculated to be $1.85 \pm 0.24$ and $0.146 \pm 0.025$, respectively. The fraction of the POPG REVs in the solution $F_{\text {vesicle }}$ was determined to be $0.074 \pm 0.015$. Because the REVs were much brighter than Rho 6G, they dominated the autocorrelation function 
Table 1. FCS Calibration Values of Rho 6G and the Different Vesicles Used in Experiments (Laser Power: 100 MW, Except Specified)

\begin{tabular}{|c|c|c|c|c|c|}
\hline & $\tau_{\text {dye }}[\mu \mathbf{s}]$ & $\tau_{\text {vesicle }}[\mathbf{m s}]$ & $\mathbf{F}_{\text {vesicle }}$ & $\mathbf{c p s}_{\text {dye }}[\mathbf{k H z}]$ & $\mathbf{c p s}_{\text {vesicle }}[\mathbf{k H z}]$ \\
\hline \hline Rho 6G & $51.5 \pm 0.8$ & - & - & 60 & - \\
\hline POPG REV & $44.5 \pm 30.3$ & $4.36 \pm 0.61$ & $0.074 \pm 0.015$ & - & 60 \\
\hline POPG RLV & - & $4.81 \pm 0.35$ & - & 60 & $958(40 \mathrm{MW})$ \\
\hline DPPG REV & $44.1 \pm 5.6$ & $2.89 \pm 0.43$ & $0.15 \pm 0.02$ & - & 228 \\
\hline DPPG RLV & - & $4.47 \pm 1.38$ & - & & $910(40 \mathrm{MW})$ \\
\hline
\end{tabular}

(the contribution of a particle to the ACF is proportional to the square of its fluorescence yield, equation 4). The overall count rate for solutions of REVs was $150 \mathrm{kHz}$ for POPG and $100 \mathrm{kHz}$ for DPPG. After addition of Triton X-100 (0.05\%) the count rates increased to $890 \mathrm{kHz}$ and $269 \mathrm{kHz}$, respectively. The $\tau_{\mathrm{D}}$ was found to be around 175-210 $\mu \mathrm{s}$, larger than the $\tau_{\mathrm{D}}$ of Rho $6 \mathrm{G}$, indicating the presence of Triton X100 micelles in solution.

\section{Antimicrobial Peptides Induce Leakage of REVs}

The interaction of antimicrobial peptides with lipid bilayers can lead to pore formation or vesicle disruption which can be tested by leakage experiments of REVs. In the case of pore formation or vesicle disruption we expect an increase of free Rho $6 \mathrm{G}$ in solution, a decrease of the number of vesicles, and an increase in the total photon count rate due to dequenching of the released fluorophore.

The interaction of magainin 2 with POPG REVs is shown in Fig. (2) where we depict the experimental ACFs to show the change in amplitude and thus in $N$ in Fig. $(\mathbf{2 A})$, and the normalized ACFs to emphasize the shift in diffusion time $\tau_{\mathrm{D}}$ with peptide addition in Fig. (2B). The interaction of melittin, polymyxin B and V4 with POPG REVs is shown in the supplement. The ACF parameters at the lowest $\mathrm{P} / \mathrm{L}$ ratio for four different antimicrobial peptides at which maximum leakage is observed are given in Table 2 . The four peptides showed overall similar effects. After addition of peptides to the vesicle solution the number of Rho $6 \mathrm{G}$ molecules, $N_{\text {rho }}$, increases while the number of REVs, $N_{\text {vesicle }}$, decreases, leading in turn to a decrease in the fraction of vesicles, $\mathrm{F}_{\text {vesicle, }}$ in the solution. Concomitantly, the photon count rate from the solution increases (Fig. 3). While for all peptides a decrease in $N_{\text {vesicle }}$ can already be seen at $\mathrm{P} / \mathrm{L}=1 / 40-1 / 20$, the total disappearance of vesicles $\left(N_{\text {vesicle }}=0\right)$ is seen only at $\mathrm{P} / \mathrm{L}=1 / 8$ - 1/4 for magainin 2 , melittin and polymyxin $\mathrm{B}$, and $\mathrm{P} / \mathrm{L}=1 / 2$ for V4. For magainin 2 and melittin the maximum increase in photon count rate was $60-70 \%$ of the value seen after Triton X-100 treatment. For polymyxin B the maximum photon count rate was around $30-40 \%$ of that observed after Triton $\mathrm{X}-100$ treatment. For V4 the maximum increase in photon count rate was $7-20 \%$.

For DPPG REVs similar results are obtained (Fig. 4). ACFs are shown in the supplement. Changes in all parameters of the ACF are seen already at $\mathrm{P} / \mathrm{L}=1 / 40$ but the total disappearance of vesicles $\left(N_{\text {vesicle }}=0\right)$ is seen at $\mathrm{P} / \mathrm{L}=1 / 4$ for magainin 2 , at $\mathrm{P} / \mathrm{L}=1 / 8-1 / 4$ for melittin, at $\mathrm{P} / \mathrm{L}=1 / 8$ for $p o-$ lymyxin $\mathrm{B}$, and at $\mathrm{P} / \mathrm{L}=1 / 4-1 / 2$ for $\mathrm{V} 4$. For magainin 2 and melittin the maximum increase in photon count rate was more than $100 \%$ of the value seen after Triton X-100 treatment, implying complete leakage. For V4 and polymyxin B this value was around $80-90 \%$. These experiments demonstrate that all peptides lead to different degrees of vesicle leakage at peptide specific $\mathrm{P} / \mathrm{L}$ ratios for both $\mathrm{POPG}$ and DPPG vesicles.

Table 2. FCS Parameters After Maximum Leakage as Induced by the Different Peptides

\begin{tabular}{|c|c|c|c|c|}
\hline & $\mathbf{P} / \mathbf{L}$ & $\tau_{\mathrm{dye}}[\mu \mathrm{s}]$ & $\tau_{\text {vesicle }}[\mathrm{ms}]$ & $\mathbf{F}_{\text {vesicle }}$ \\
\hline \multicolumn{5}{|c|}{ POPG REV } \\
\hline Triton X-100 & - & $175 \pm 2$ & - & - \\
\hline Magainin 2 & $1 / 4$ & $53.9 \pm 0.7$ & $5.43 \pm 1.03$ & $0.0013 \pm 0.00028$ \\
\hline Melittin & $1 / 4$ & $51.4 \pm 0.7$ & - & - \\
\hline Polymyxin B & $1 / 4$ & $52.7 \pm 1.1$ & - & - \\
\hline V4 & $1 / 2$ & $54.3 \pm 0.4$ & - & - \\
\hline \multicolumn{5}{|c|}{ POPG RLV } \\
\hline Triton X-100 & & $389 \pm 7$ & - & - \\
\hline Magainin 2 & $1 / 2$ & - & $1.13 \pm 0.18$ & - \\
\hline Melittin & $1 / 2.67$ & $55.4 \pm 20.9$ & - & - \\
\hline Polymyxin B & $1 / 4$ & $49.2 \pm 2.2$ & - & - \\
\hline V4 & $1 / 2$ & $61.4 \pm 8.2$ & - & - \\
\hline \multicolumn{5}{|c|}{ DPPG REV } \\
\hline Triton X-100 & - & $208 \pm 4$ & - & - \\
\hline Magainin 2 & $1 / 4$ & $50.5 \pm 0.6$ & $3.31 \pm 1.05$ & $0.0032 \pm 0.0016$ \\
\hline Melittin & $1 / 4$ & $52.6 \pm 0.8$ & - & - \\
\hline Polymyxin B & $1 / 4$ & $49.9 \pm 0.8$ & - & - \\
\hline V4 & $1 / 4$ & $52.3 \pm 0.7$ & - & - \\
\hline \multicolumn{5}{|c|}{ DPPG RLV } \\
\hline Triton X-100 & - & $374 \pm 8$ & - & - \\
\hline Magainin 2 & $1 / 2$ & - & $4.86 \pm 1.10$ & - \\
\hline Melittin & $1 / 2.67$ & $370 \pm 87$ & - & - \\
\hline Polymyxin B & $1 / 4$ & $56.4 \pm 7.4$ & - & - \\
\hline V4 & $1 / 4$ & $49.3 \pm 10.5$ & - & - \\
\hline
\end{tabular}



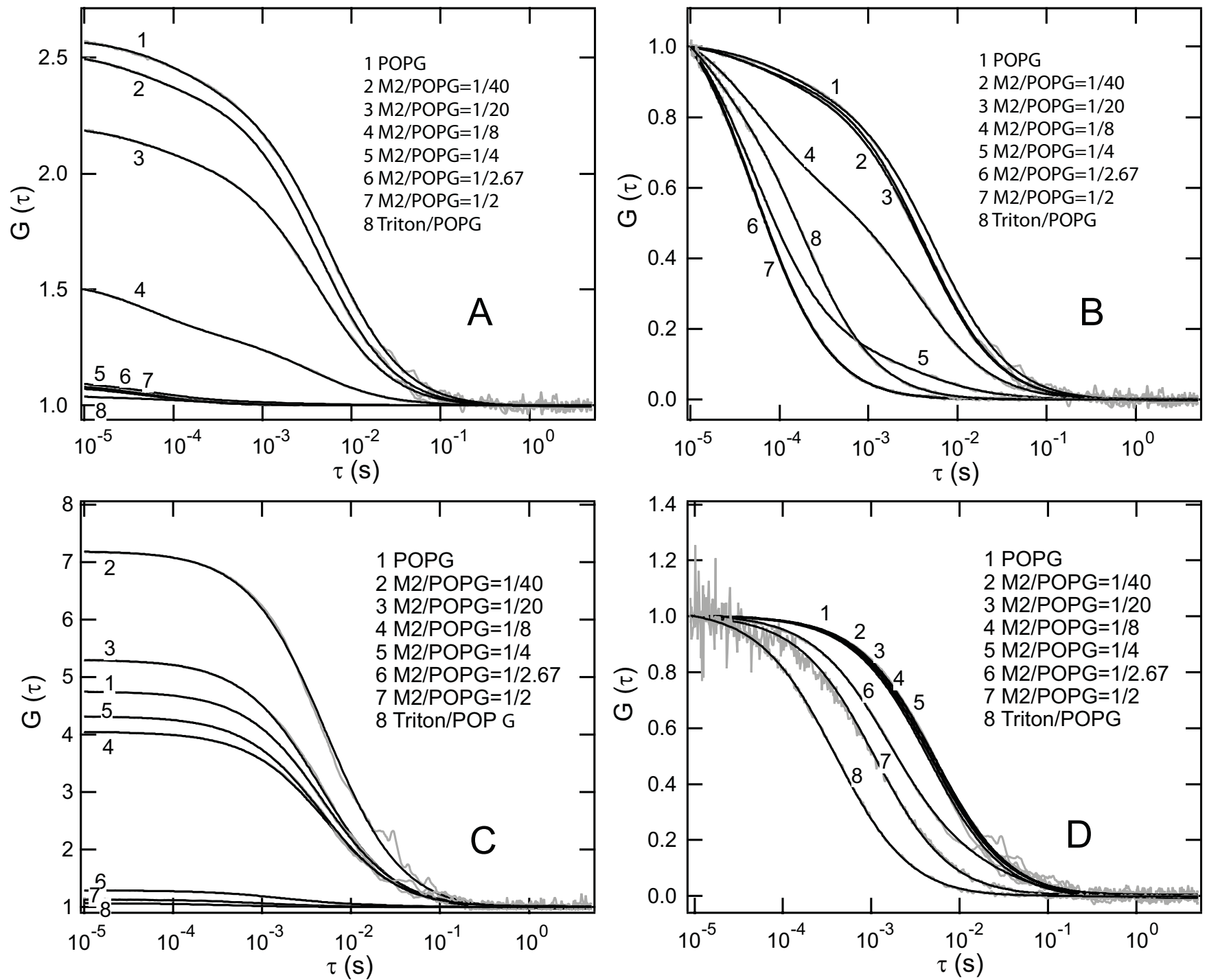

Fig. (2). Interaction of magainin 2 with POPG REVs and RLVs. (A) and (B) demonstrate the interaction of magainin 2 with POPG REVs. (A) is the measured autocorrelation functions and their fits where the change of amplitude can be clearly seen with increasing peptide concentration. (B) depicts the normalized autocorrelation functions in which the change of width is easier to see. (C) and (D) show the interaction of magainin 2 with POPG RLVs. (C) is the measured autocorrelation functions and their fits. (D) depicts the normalized autocorrelation functions.

\section{Antimicrobial Peptides Interact with RLVs}

Many antimicrobial peptides are reported to aggregate or disrupt bacteria and vesicles [50]. RLVs can be used to test these cases. It should be noted that aggregation and disruption both will lead to a range of particle sizes with very different cps making quantification with FCS difficult due to the non-linear contribution to the ACF by particles of different cps. We thus use only the apparent number of particles $N_{a p p}$ (Eq. 4) to characterize the solutions. If aggregation is induced the particles should grow and $N_{a p p}$ should decrease with a concomitant increase of $\tau_{\mathrm{D}}$. In the case of vesicle disruption $N_{a p p}$ should increase and $\tau_{\mathrm{D}}$ should accordingly decrease. In the case of pore formation only, no changes should be seen.

The ACFs of POPG RLVs after addition of different concentrations of magainin 2 are shown in Fig. (2), where we depict the experimental ACFs to show the change in amplitude and thus in $N_{a p p}$ in Fig. (2C), and the normalized ACFs to emphasize the shift in diffusion time with peptide addition in Fig. (2D). The interaction of melittin, polymyxin B and V4 with POPG RLVs are shown in the supplement. In Fig. (5) the change of the ACF fit parameters is shown in dependence of peptide concentration. With addition of peptides to the POPG RLVs some general trends can be observed. Melittin, polymyxin B and V4, show a decrease in $N_{a p p}$ and an increase in $\tau_{\mathrm{D}}$ at smaller $\mathrm{P} / \mathrm{L}$ ratios (melittin: $\mathrm{P} / \mathrm{L}$ $\leq 1 / 4$; polymyxin $\mathrm{B}: \mathrm{P} / \mathrm{L} \leq 1 / 20 ; \mathrm{V} 4: \mathrm{P} / \mathrm{L} \leq 1 / 2.67$ ) indicating aggregation. Magainin 2 does not show a similar trend and $N_{a p p}$ and $\tau_{\mathrm{D}}$ stay approximately constant for $\mathrm{P} / \mathrm{L} \leq 1 / 4$. At higher, peptide specific $\mathrm{P} / \mathrm{L}$ ratios all peptides lead to a strong increase in $N_{a p p}$ and a strong decrease of $\tau_{\mathrm{D}}$, indicating vesicle disruption. 

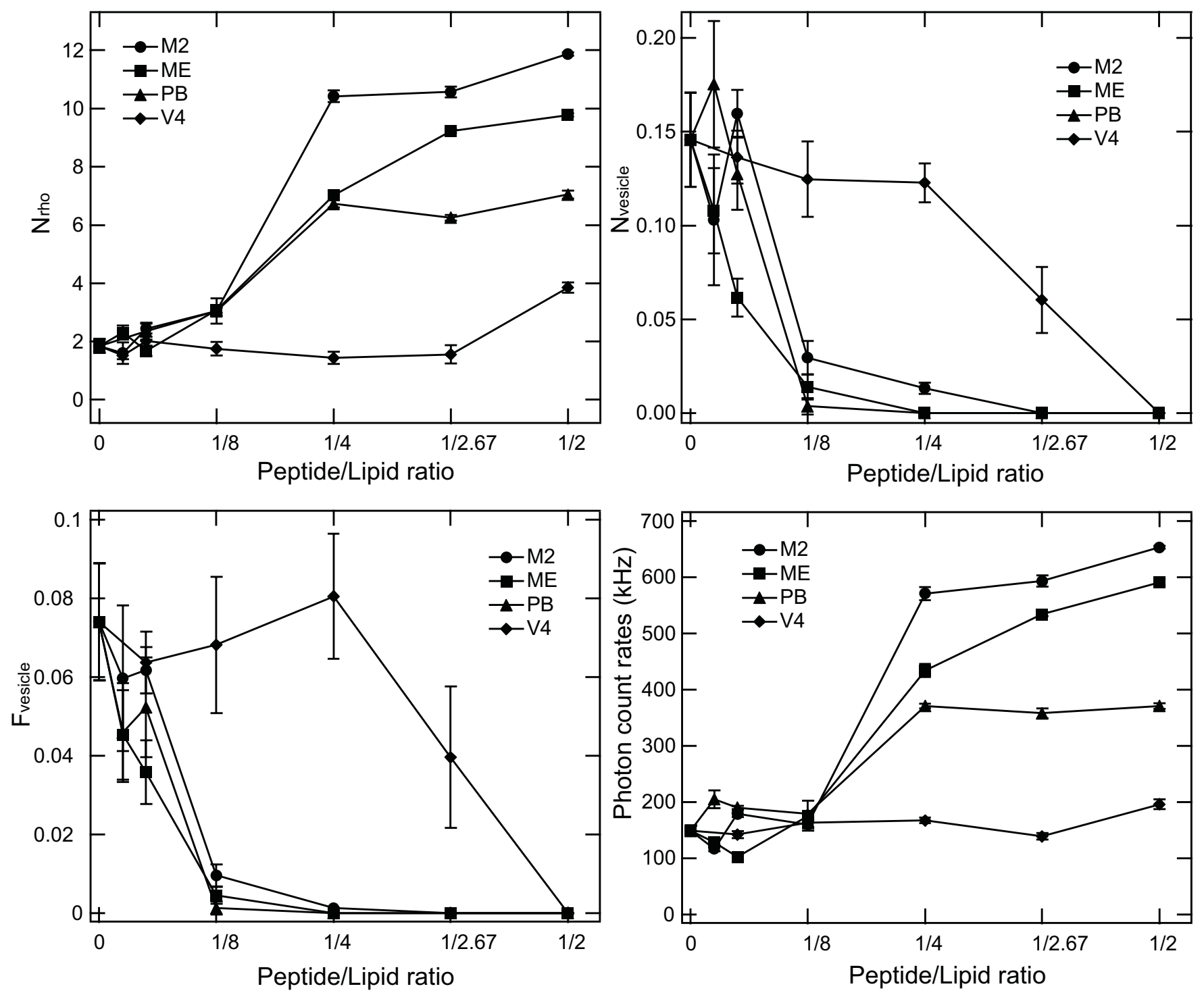

Fig. (3). Comparison of $N_{\text {rho }}, N_{\text {vesicle }}, F_{\text {vesicle }}$ and photon count rates of antimicrobial peptides interacting with POPG REVs.

The data for the interaction between the four peptides and DPPG RLVs are shown in Fig. (6). In general similar trends are seen as well for the POPG RLVs with some marked differences. Within the concentration range used, magainin 2 does not show any changes in $N_{a p p}$ or $\tau_{\mathrm{D}}$ indicating that no vesicle disruption took place. Melittin and V4 show vesicle disruption at smaller $\mathrm{P} / \mathrm{L}$ ratios while polymyxin $\mathrm{B}$ shows no difference.

As control experiments we used POPC RLVs. As shown in Fig. (7), no changes are seen for any of the peptides in the diffusion time $\tau_{\mathrm{D}}$ contrary to the other experiments on labeled POPG and DPPG vesicles.

The apparent aggregation of POPG (Fig. 8) and DPPG (data not shown) vesicles is supported for melittin, polymyxin B and V4 by confocal images while for magainin 2 no aggregates are found.

\section{DISCUSSION}

A combination of leakage measurements on REVs and measurements of RLVs were conducted to investigate whether antimicrobial peptides aggregate, disrupt and/or render lipid vesicles permeable [29]. If the antimicrobial peptides form pores on the membrane, a decrease in diffusion time for REVs and a constant diffusion time for RVLs will be found. However, if the antimicrobial peptides disrupt the membrane in a detergent-like manner, a decrease in diffusion time for both REVs and RLVs will be detected (Fig. 1). In this study we used POPG and DPPG because of their negative charge as models for bacterial membranes and to test whether the saturation of the lipid tails has any influence on the activity of the peptides.

\section{Magainin 2}

It is believed that magainin 2 induces membrane permeation through the formation of a toroidal pore $[28,51]$. Our data suggest that magainin 2 induces pore formation to cause 

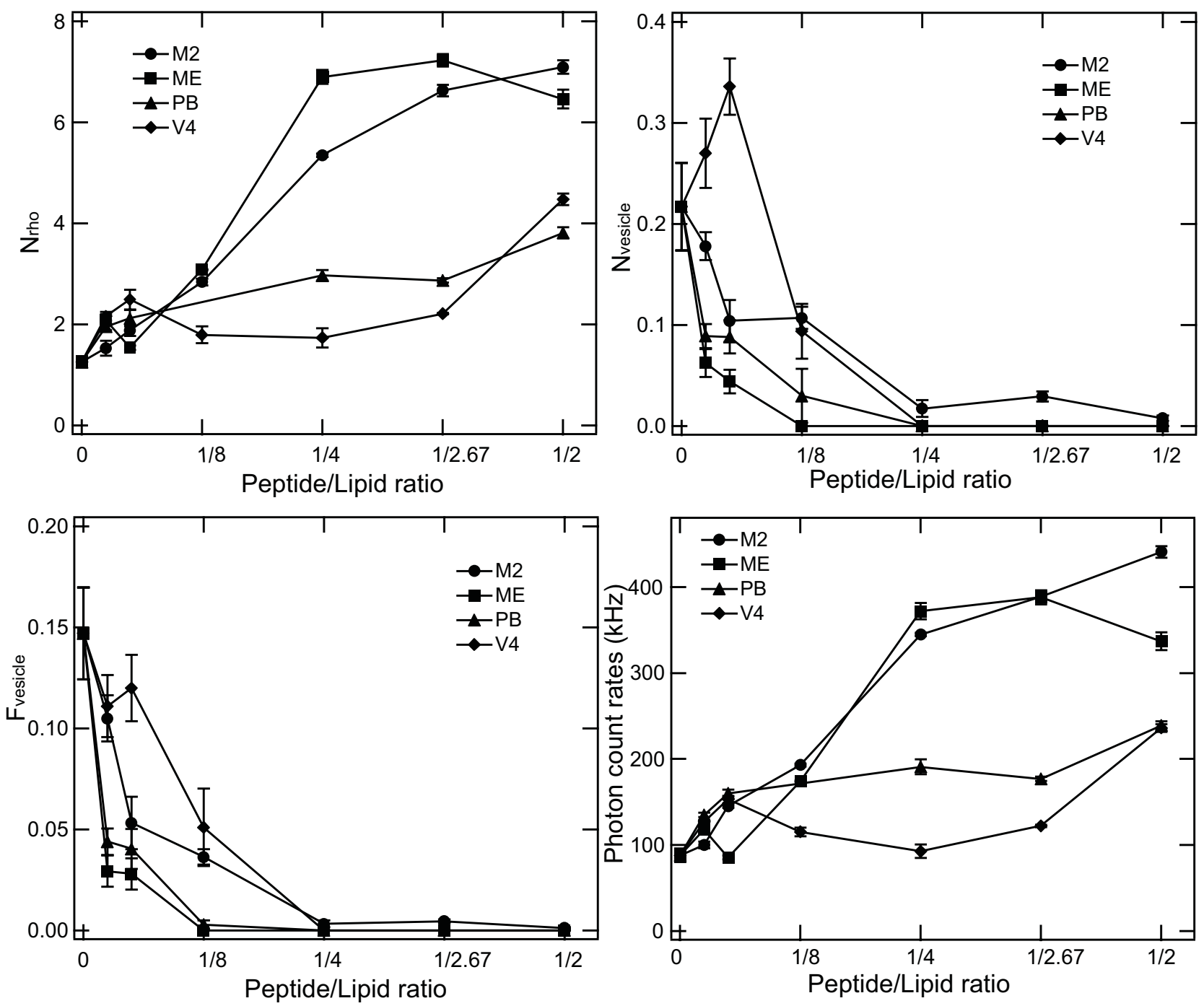

Fig. (4). Comparison of $N_{r h o}, N_{\text {vesicle }}, F_{\text {vesicle }}$ and photon count rates of antimicrobial peptides interacting with DPPG REVs.

membrane permeation. In this study magainin 2 induced complete leakage of POPG REVs at a P/L of $1 / 4$ with an increase of the count rate up to $60-70 \%$ of the count rate seen after Triton X-100 addition (Fig. 3). However at $\mathrm{P} / \mathrm{L} \leq 1 / 4$, neither a size change in the REVs nor a size change of the POPG RLVs could be observed and thus magainin 2 does not lead to aggregation of vesicles (Figs. 3,5). This is confirmed by the confocal images of magainin $2 /$ RLV solutions where no aggregates could be observed (Fig. 8). We therefore suggest that magainin 2 induces vesicle leakage by pore formation at low $\mathrm{P} / \mathrm{L}$ ratios until complete leakage is observed at $\mathrm{P} / \mathrm{L} \sim 1 / 4$. For $\mathrm{P} / \mathrm{L}>1 / 4$ magainin 2 induced membrane destabilization, leading to membrane disruption indicated by a decrease in the size of the vesicles. However, no aggregation can be seen at any peptide concentration tested. DPPG experiments paint a similar picture of magainin 2 action, with the same $\mathrm{P} / \mathrm{L}$ ratio for complete vesicle leakage, and a lack of vesicle aggregation (Figs. 4,6). But in contrast to POPG, DPPG vesicles are never destabilized and no membrane disruption is seen at the concentrations tested.
The measurements thus show that magainin 2 induces leakage by pore formation without vesicle aggregation possibly followed by disruption at a much higher concentration.

\section{Melittin}

Melittin is known to form pores on the membrane and lead to the death of the target bacteria $[27,52]$. The present study showed that melittin also induces pores to perform its function. From P/L ratios of 1/40 onwards changes in $N_{\text {vesicle }}$ and the vesicle fraction $\mathrm{F}_{\text {vesicle }}$ show the beginning leakage which is complete at a ratio of $1 / 4$ for POPG REVs, with an increase of the count rate up to $60-70 \%$ of the count rate seen after Triton X-100 addition (Fig. 3). However, no particle smaller than POPG vesicles was detected in the experiment of POPG RLVs in the presence of melittin, which suggests that POPG vesicles were not disrupted (Fig. 5). Instead, pores were formed at these $\mathrm{P} / \mathrm{L}$ ratios, leading to membrane permeation. When the $\mathrm{P} / \mathrm{L}$ ratio increased to $1: 2.67$ and higher, smaller aggregates were found as shown by the decrease in diffusion time (Fig. 5). In contrast to magainin 2, 

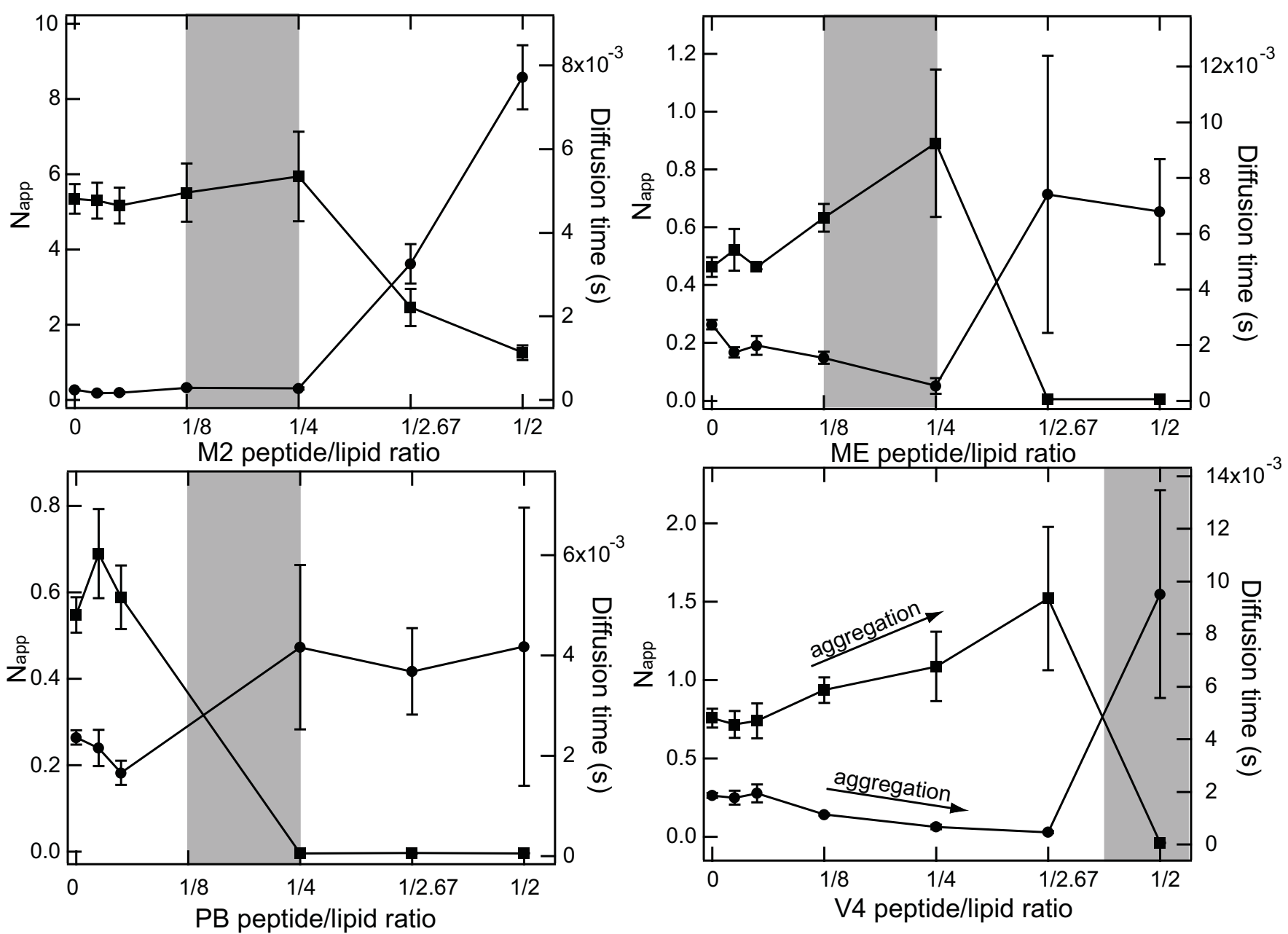

Fig. (5). Comparison of $N_{a p p}$ and diffusion time of antimicrobial peptides interacting with POPG RLVs ( $\bullet$ diffusion time; $\left.\bullet N_{a p p}\right)$. The P/L ranges in which full leakage are observed are marked in grey.

melittin promoted aggregation during the process of inducing vesicle permeation. Melittin induced slight aggregation of POPG RLVs at a P/L ratio of $1: 8$, and at 1:4, the aggregates increased in size (Fig. 5). The aggregation is confirmed by confocal images (Fig. 8). For DPPG vesicle measurements full leakage is as well observed for $\mathrm{P} / \mathrm{L}=1 / 4$, below which only partial leakage and vesicle aggregation is seen (Fig. 4). In this case disruption and leakage of vesicles happens at the same $\mathrm{P} / \mathrm{L}$ ratios between $1 / 4$ and $1 / 8$ (Figs. 4,6). Whereas leakage happens clearly before disruption of the POPG vesicles, in DPPG vesicles full leakage happens either only slightly before or even at the same time as vesicle disruption. We therefore suggest that for melittin pore formation happens in conjunction with some aggregation and possibly disruption.

\section{Polymyxin B}

Polymyxin B is an antimicrobial peptide similar to melittin, with high toxicity. Current research focuses on the interaction between polymyxin B and the outer bacterial membrane in which LPS plays an important role [17, 19, 20]. Little is known about the fate of the inner membrane. This study demonstrates that polymyxin B disrupts the membrane at a $\mathrm{P} / \mathrm{L}$ ratio of 1:8 with an increase of the count rate up to
$40 \%$ of the count rate seen after Triton X-100 addition (Fig. 3 ). The great variance in the diffusion time (from $1.6 \mathrm{~ms}$ to 2 s) indicated the presence of both fragments and aggregates of the vesicles in solution. The fragments probably coalesced to form large aggregates. This was corroborated by the measurements at increased $\mathrm{P} / \mathrm{L}$ ratios of 1:4, 1:2.67 and 1:2. Excluding large aggregates, a decrease in the diffusion time of POPG RLVs was detected, possibly representing vesicle fragments (Fig. 5). Correspondingly, at these ratios polymyxin B induced maximum leakage from POPG REVs. However, the increase of the count rate to only $40 \%$ of the expected value, as tested with Triton X-100, might indicate that remaining vesicle aggregates still contain some unreleased Rho 6G. In the case of DPPG vesicles the same behaviour can be seen and no influence of the tail groups on the action of polymyxin B is detectable (Fig. 6). Taken together, the results suggest that polymyxin B adopts the carpet mechanism to cause membrane permeation without pore formation.

\section{V4}

$\mathrm{V} 4$ did not induce membrane permeation until a $\mathrm{P} / \mathrm{L}$ ratio of $1: 2$. However, the membrane did change at $\mathrm{P} / \mathrm{L}$ ratios lower than 1:2 (Fig. 3). At P/L of 1:8 and 1:4, V4 induced a 

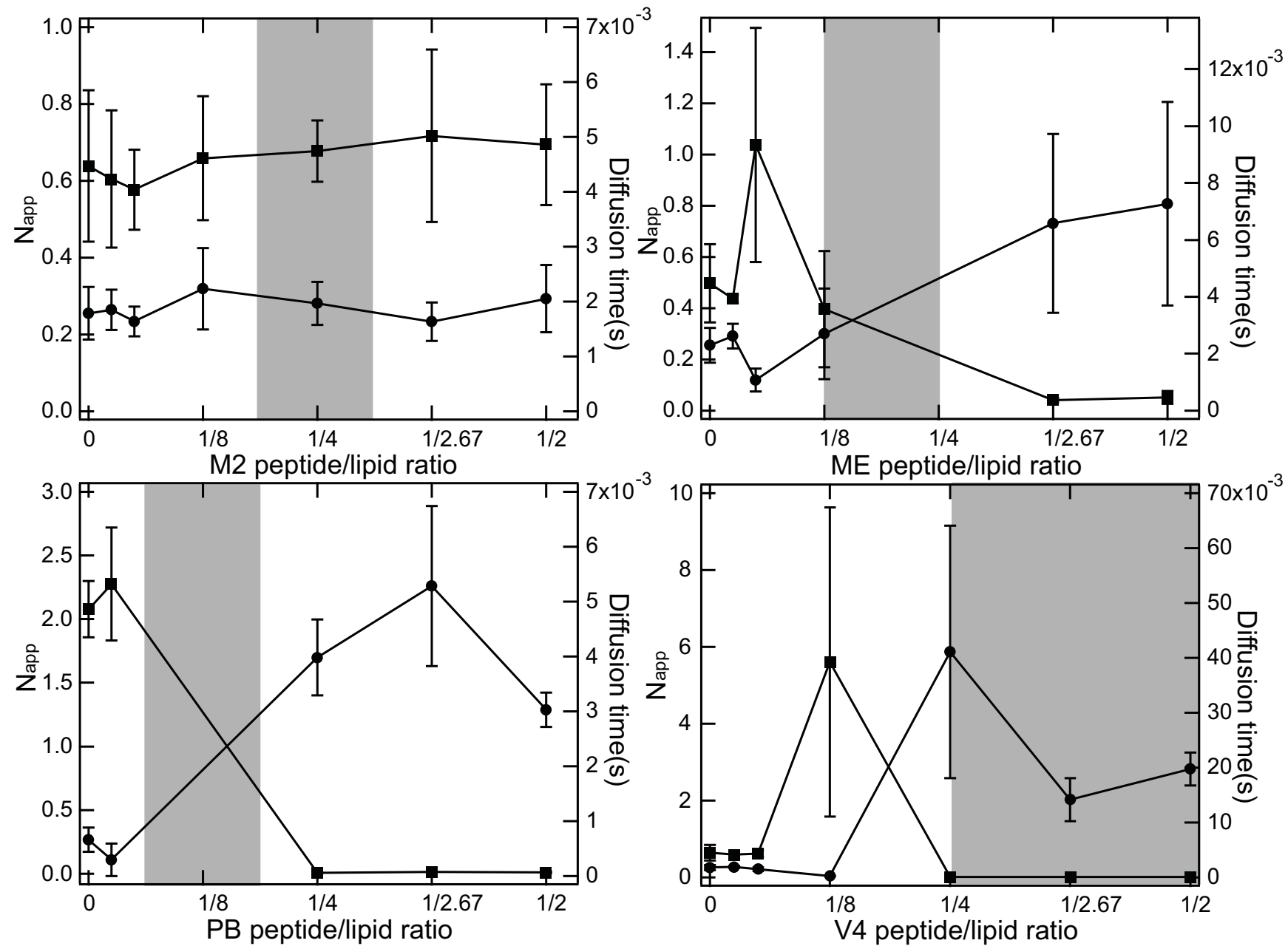

Fig. (6). Comparison of $N_{a p p}$ and diffusion time of antimicrobial peptides interacting with DPPG RLVs (๘ diffusion time; $\left.\bullet N_{a p p}\right)$ The P/L ranges in which full leakage are observed are marked in grey.

small increase in the diffusion time of the POPG RLVs (Fig. 5). At a $\mathrm{P} / \mathrm{L}$ ratio of $1: 2.67$, the increase was more apparent and the diffusion time reached about twice the value of POPG RLVs. Correspondingly, the particle number in the confocal volume decreased when the $\mathrm{P} / \mathrm{L}$ ratio was increased from 1:8 to 1:2.67. These results indicate that V4 induces aggregation of POPG vesicles but without leakage. When the $\mathrm{P} / \mathrm{L}$ ratio increased to $1: 2$, the diffusion time of POPG RLVs decreased strongly with a concomitant increase in $N_{a p p}$. Therefore, V4 induces membrane permeation at this ratio but with an increase of the count rate of up to only $6-20 \%$ of the count rate seen after Triton X-100 addition. It should be noted that except for the fragments of the vesicles at a $\mathrm{P} / \mathrm{L}$ ratio of $1: 2$, there were also large aggregates which might be the result of coalesced POPG vesicle fragments after V4 induced vesicle disruption. In the case of DPPG full vesicle leakage is already seen at a $\mathrm{P} / \mathrm{L}$ ratio of $1 / 4$ with an increase of the count rate up to $80 \%$ of the count rate seen after Triton X-100 addition (Fig. 4). The low release of Rho 6G compared to the other investigated peptides might be attributed to the entrapment of Rho $6 \mathrm{G}$ in the large aggregates seen. Similar to polymyxin B, no pore formation was detected.

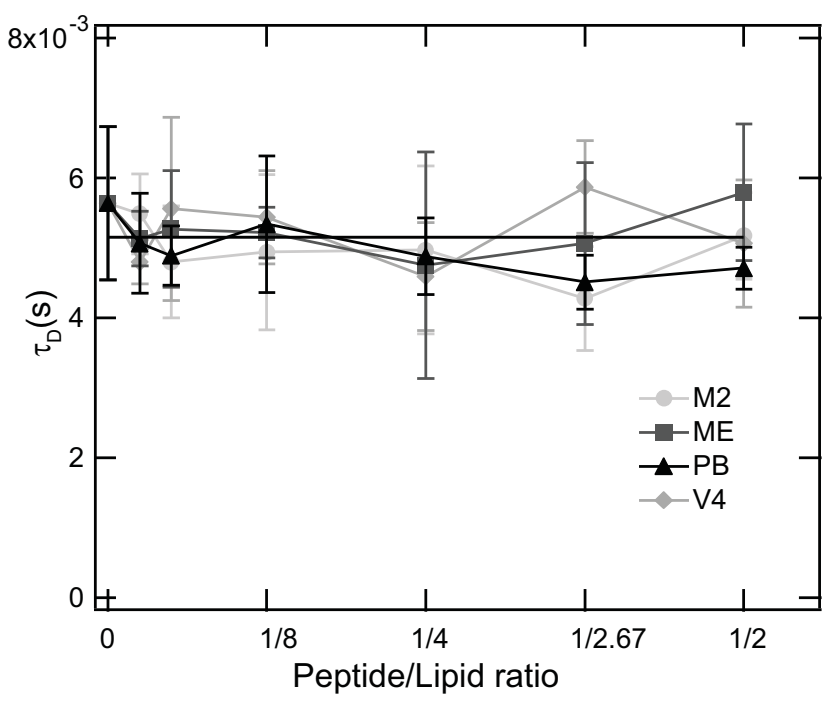

Fig. (7). Comparison of diffusion time of antimicrobial peptides interacting with POPC RLVs. No disruption has been observed.

V4 is a highly hydrophobic antimicrobial peptide and even aggregated in the buffer itself because of the high hydrophobic interaction between the peptide molecules [24]. 
Different from polymyxin B, membrane aggregation is promoted by V4 before membrane disruption. In combination with the lower release of Rho 6G this might indicate an action mechanism by aggregation (agglutination of bacteria), possibly fusion, and permeation.

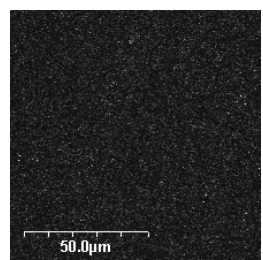

POPG

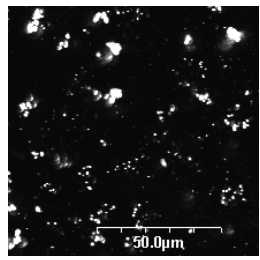

POPG \&PB

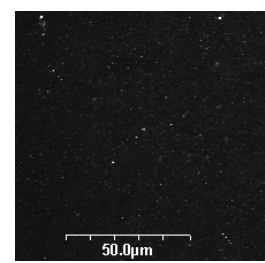

POPG\&M2

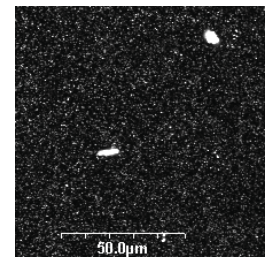

POPG\&V4

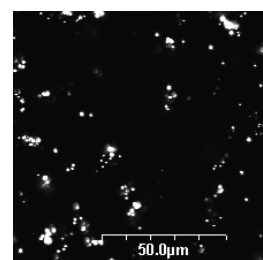

POPG\&ME
Fig. (8). Confocal images of POPG RLVs in the absence and presence of antimicrobial peptides. (Final concentration of lipids: 40 $\mu \mathrm{M}$; final peptide/lipid ratio: magainin 2 , 1:2; melittin, 1:4; polymyxin B, 1:8; V4, 1:4).

\section{POPG Versus DPPG Vesicles}

Lipids can have an influence on the mechanism of antimicrobial peptides due to their different head group types and/or the length and saturation of the tail groups. The investigated peptides showed at the tested concentrations and $\mathrm{P} / \mathrm{L}$ ratios actions only on negatively charged lipids. However, the influence of the tail groups is less clearly characterized. We therefore used saturated DPPG and unsaturated POPG lipid vesicles to test if the degree of saturation has any influence on the peptide-membrane interactions. The hypothesis in our case was that the saturated DPPG, forming a more densely packed layer, hinders the interaction of the antimicrobial peptides with the hydrophobic core of the lipid bilayer. In the case of magainin 2 this seemed to be confirmed. Although magainin 2 induces vesicle leakage at the same $\mathrm{P} / \mathrm{L}$ ratio for POPG and DPPG, DPPG vesicles, in contrast to POPG vesicles, could not be disrupted at the same concentrations used for magainin 2 (Figs. 5,6). For melittin almost no differences between the two lipids could be discerned in respect to leakage and membrane permeation/disruption. However, as shown in the ACFs in the supplement, more and larger aggregates can be seen. For polymyxin B no differences between the two lipids could be detected. For V4 vesicle aggregation was much more pronounced for DPPG vesicles and leakage and disruption were observed earlier than for POPG vesicles. Thus the influence of the lipid tail groups on antimicrobial function is strongly peptide dependent. While for magainin 2 disruption of the more densely packed DPPG vesicles is delayed, the saturation of the lipid tails had no influence on polymyxin B. For melittin and V4 the more densely packed DPPG membrane presumably lead to a weaker interaction of the peptides with the hydrophobic core, a larger concentration of the peptides on the surface, and thus a stronger peptide driven aggregation of the vesicles.

\section{Difference in Mechanism for Four Antimicrobial Pep- tides}

Although all the studied antimicrobial peptides finally disrupt the membrane, adopting the carpet mechanism or a modified carpet mechanism to perform their function, there were apparently some differences between them (Fig. 9). Firstly, before the vesicles were disrupted, magainin 2 and melittin formed pores in the vesicle membranes, which were detected at a certain intermediate $\mathrm{P} / \mathrm{L}$ ratio. However, polymyxin B and V4 disrupted the membrane directly with no detectable pore formation. This difference might result from the structure of the peptides. It is known that magainin 2 and

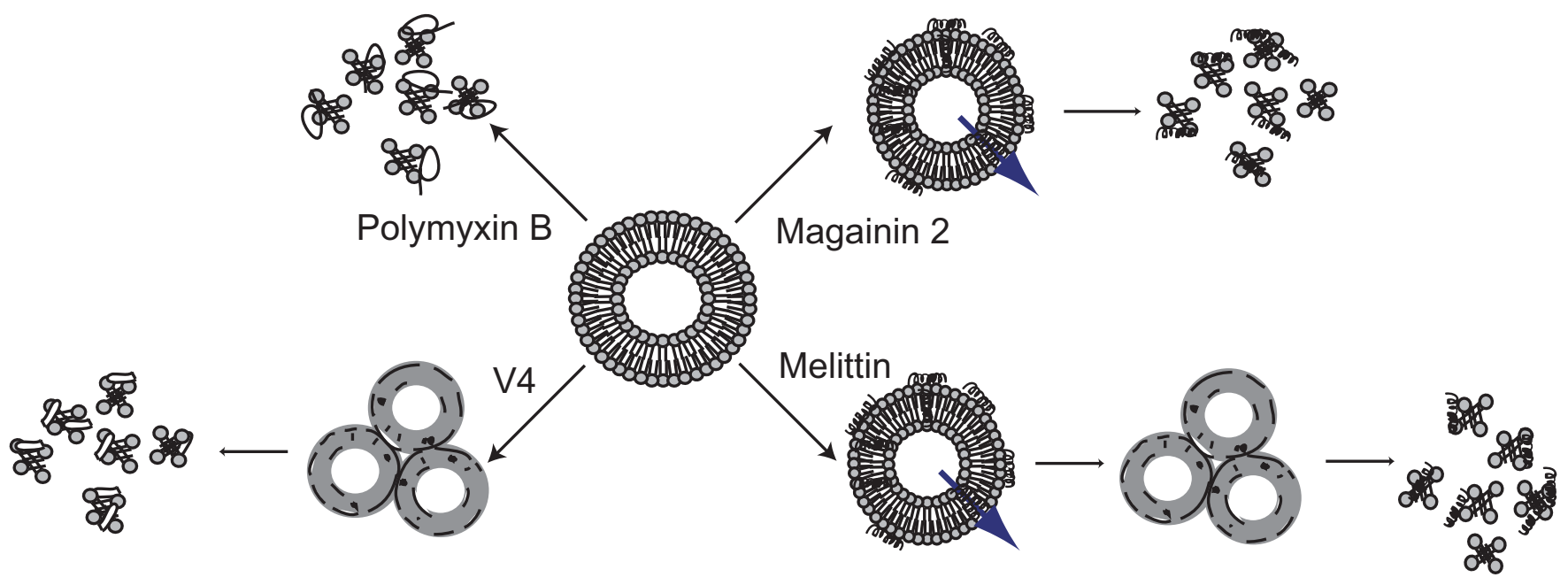

Fig. (9). Comparison of mechanisms of antimicrobial peptides inducing membrane permeation. With the increasing peptide/lipid ratio, magainin 2 induced pore formation and then membrane disruption. No aggregation was detected. Melittin first induced pore formation as well as membrane aggregation. When peptide/lipid ratio further increased, membrane was disrupted. Polymyxin B directly caused membrane disruption. V4 first induced membrane aggregation but no membrane permeation. With the increasing peptide/lipid ratio, membrane disruption happened. 
melittin form $\alpha$-helices upon interaction with lipid membranes. The amphipathic structure of the peptide makes it possible that spatially separate hydrophobic and hydrophilic faces perform different functions. The hydrophobic face interacted with the acyl chains of the phospholipids. Correspondingly, the hydrophilic face not only interacted with the head groups of the phospholipids through electrostatic interaction but also pointed inwards to form pores. When the P/L ratio was further increased, the peptides penetrate the inner layer of the vesicle, destabilize the membrane and finally cause membrane disruption at high concentrations. Different from the two $\alpha$-helical antimicrobial peptides investigated, polymyxin B and V4 are cyclic peptides which have a relatively rigid structure. It is difficult for these cyclic peptides to change the conformation and form a similar structure to $\alpha$ helical peptides. Therefore, polymyxin B and V4 possibly act in a detergent-like manner to disrupt the membrane.

The differential propensity to aggregate distinguishes the four antimicrobial peptides under investigation. Within the studied range of $\mathrm{P} / \mathrm{L}$ ratios, melittin, polymyxin $\mathrm{B}$ and $\mathrm{V} 4$ showed apparent aggregation, which makes them different from magainin 2. Whether aggregates formed or not is possibly related to hydrophobic interactions. Study of the amino acid distribution of magainin 2 in the $\alpha$-helical conformation showed that it is amphipathic [53] with some polar amino acids separating the hydrophobic face into several small hydrophobic portions, which weakened the hydrophobicity. Therefore when magainin 2 is absorbed on the surface of lipid vesicles or vesicle fragments, the hydrophobic interaction was not strong enough to bring the vesicles close together for aggregation.

The results of confocal imaging were consistent with those of FCS. Magainin 2 did not induce any aggregation in contrast to the other three antimicrobial peptides (Fig. 8). It should be noted that the concentration of large aggregates is relatively low and in FCS experiments, the large aggregates are seen only occasionally, thus explaining why in the majority of FCS measurements a homogeneous solution is observed.

We therefore suggest that the primary interaction leading to binding of antimicrobial peptides to membranes is electrostatic [24], the type of permeation, i.e. carpet mechanism or pore formation, is dependent on the type of peptide, and the sequence and $\mathrm{P} / \mathrm{L}$ ratio of peptide induced aggregation and permeation is influenced by the lipid tail groups.

The findings presented here are consistent with earlier studies which reported full vesicle leakage in a P/L range of $1 / 10-1 / 2$ for magainin 2 [54-56] and no penetration below $\mathrm{P} / \mathrm{L}=1 / 60$ and full insertion at $1 / 10[11,57]$. For melittin, leakage and stable pore formation was reported for $\mathrm{P} / \mathrm{L}=$ $1 / 10000-1 / 20$ [27, 50, 58]. Earlier studies by Pramanik et al. used the same FCS based technique to investigate the interaction of magainin and melittin with POPC REVs and RLVs to unravel their mechanisms of action [29]. Magainin and melittin were found to disrupt the membrane and form pores on the membrane, respectively, different from our findings. However the species of magainin was not specified and was possibly different from magainin 2 and the lipids were not representative of the bacterial membrane in which electrostatic interactions play a major role in peptide-lipid interactions. In addition, only one peptide/lipid ratio was presented to qualitatively study the effect of peptides on vesicles. Magzoub et al. compared several cell-penetrating peptides and found that melittin induced membrane permeation at peptide/lipid ratio of $1 / 20$, which is comparable to the result of the present study [50]. However due to the large difference in fluorescence yield, no absolute quantifications of the release of fluorophore was obtained. In this study, we extended the range of peptide/lipid ratio and resolved the problem of different fluorescence yield of particles, allowing the quantification of the peptide-lipid interaction using FCS. Simultaneously, we compared the interaction between 4 different antimicrobial peptides, representing different structures and origins, and negatively charged phospholipids, which are important components of bacterial plasma membranes. Our study provides comprehensive and comparative in vitro evidence for antimicrobial activity of these peptides against bacterial lipids.

\section{CONCLUSION}

Antimicrobial peptides have drawn an increasing interest in drug development due to their clinical potential. In this paper we applied FCS to quantitatively investigate the interaction between antimicrobial peptides (magainin 2, melittin, polymyxin B and a rationally designed peptide, V4) and artificial membranes which can function as bacterial models. We compared the mechanisms of different antimicrobial peptides inducing membrane permeation. We have shown that magainin 2 induces pore formation before membrane disruption without aggregation, whereas melittin induces pore formation, membrane aggregation and finally membrane disruption. Polymyxin B causes membrane disruption and aggregation at the same time whereas V4 induces membrane aggregation first followed by membrane disruption. This is probably due to the innate structural difference of each of these peptides which, upon their interaction with membranes, exhibit such differential antimicrobial effects. We also showed that due to the hydrophobic interaction, melittin, polymyxin B and V4 all promoted different extents of aggregation while magainin 2 did not. Magainin 2, melittin and polymyxin B showed similar activity in inducing permeation into negatively charged membranes. Considering the lower solubility, V4 which is a designed antimicrobial peptide showed strong ability to induce membrane permeation compared with the other three well-studied antimicrobial peptides, which indicated a promising future for drug development of V4. Therefore if the peptide solubility could be improved, the antimicrobial activity of V4 would be expected to increase substantially. Taken together, designing new peptides with a more hydrophilic or detergent-like structure would possibly increase the solubility and thus the activity of the peptide.

\section{ACKNOWLEDGEMENTS}

The research in this work was funded by a grant from the faculty research committee of the NUS. LY acknowledges support by a NUS graduate scholarship. 


\section{SUPPLEMENT TO MANUSCRIPT}

This supplement contains all experimental autocorrelation functions of the four antimicrobial peptides during their interactions with POPG and DPPG in the form of RLVs or REVs. The supplement consists of four figures. Figs. (3-6) in the manuscript are the summary of Fig. (2) in the manuscript and Figs. (S1-S4) in this supplement.
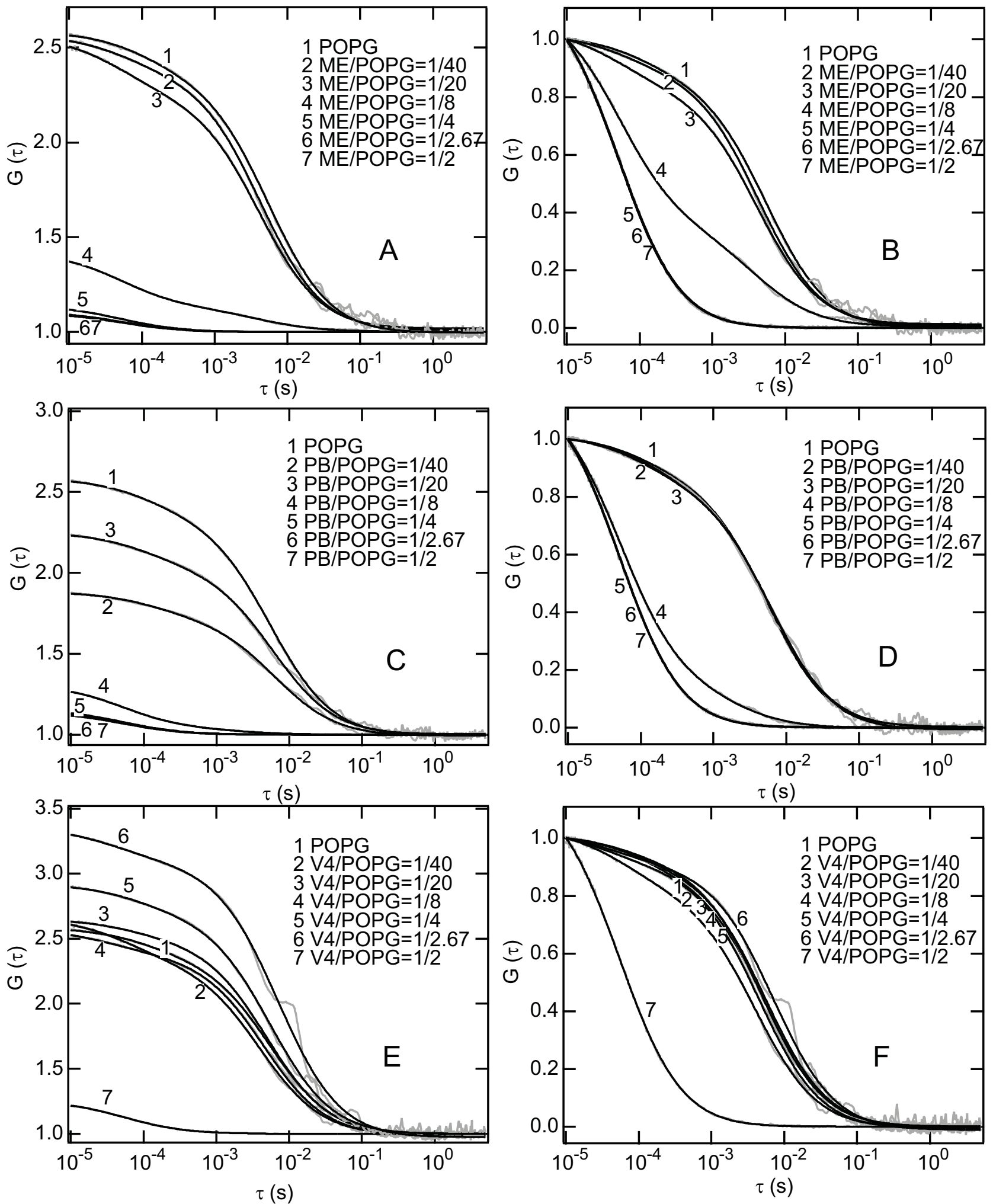

Fig. (S1). Interaction of melittin, polymyxin B and V4 with POPG REVs. The graphs on the left (A, C and E) are the measured autocorrelation functions and their fits, where the change of amplitude can be clearly seen with increasing peptide concentration. The graphs on the right (B, D and F) depict normalized autocorrelation functions, in which the change of width is easier to see. 

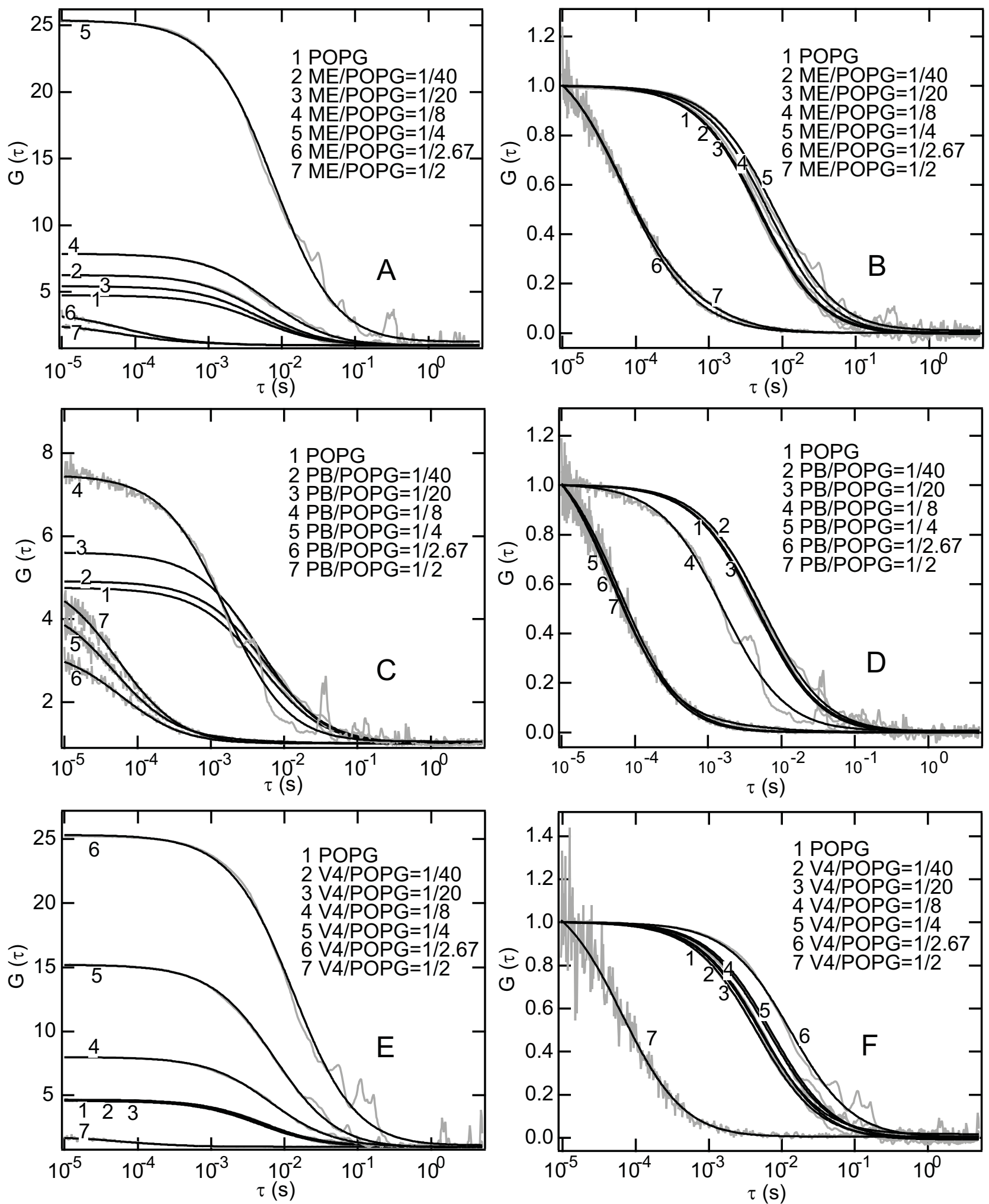

Fig. (S2). Interaction of melittin, polymyxin B and V4 with POPG RLVs. The graphs on the left (A, C and E) are the measured autocorrelation functions and their fits. The graphs on the right $(\mathbf{B}, \mathbf{D}$ and $\mathbf{F})$ depict normalized autocorrelation functions. 

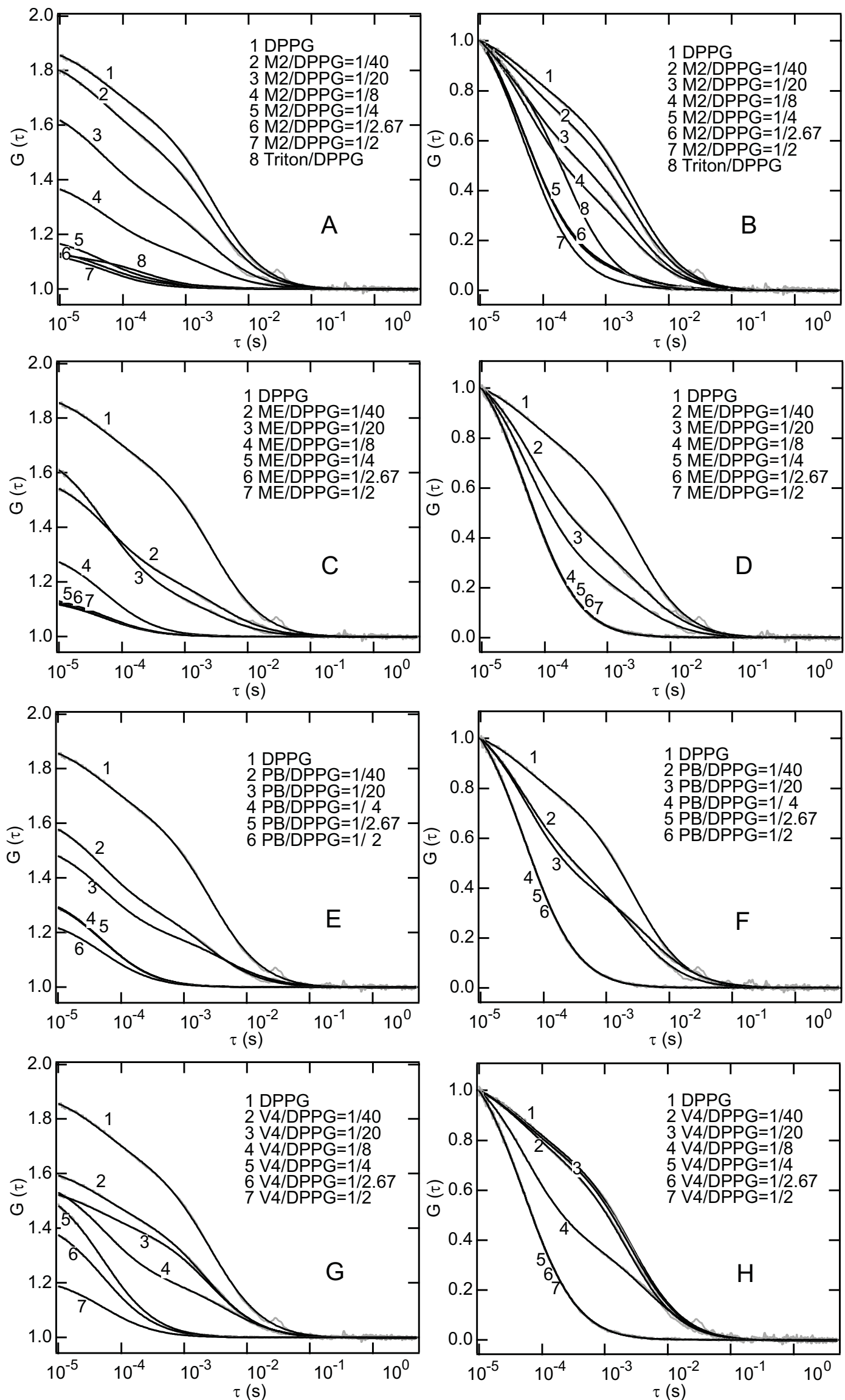

Fig. (S3). Interaction of four antimicrobial peptides with DPPG REVs. The graphs on the left (A, C, $\mathbf{E}$ and $\mathbf{G})$ are the measured autocorrelation functions and their fits. The graphs on the right $(\mathbf{B}, \mathbf{D}, \mathbf{F}$ and $\mathbf{H})$ depict normalized autocorrelation functions. 

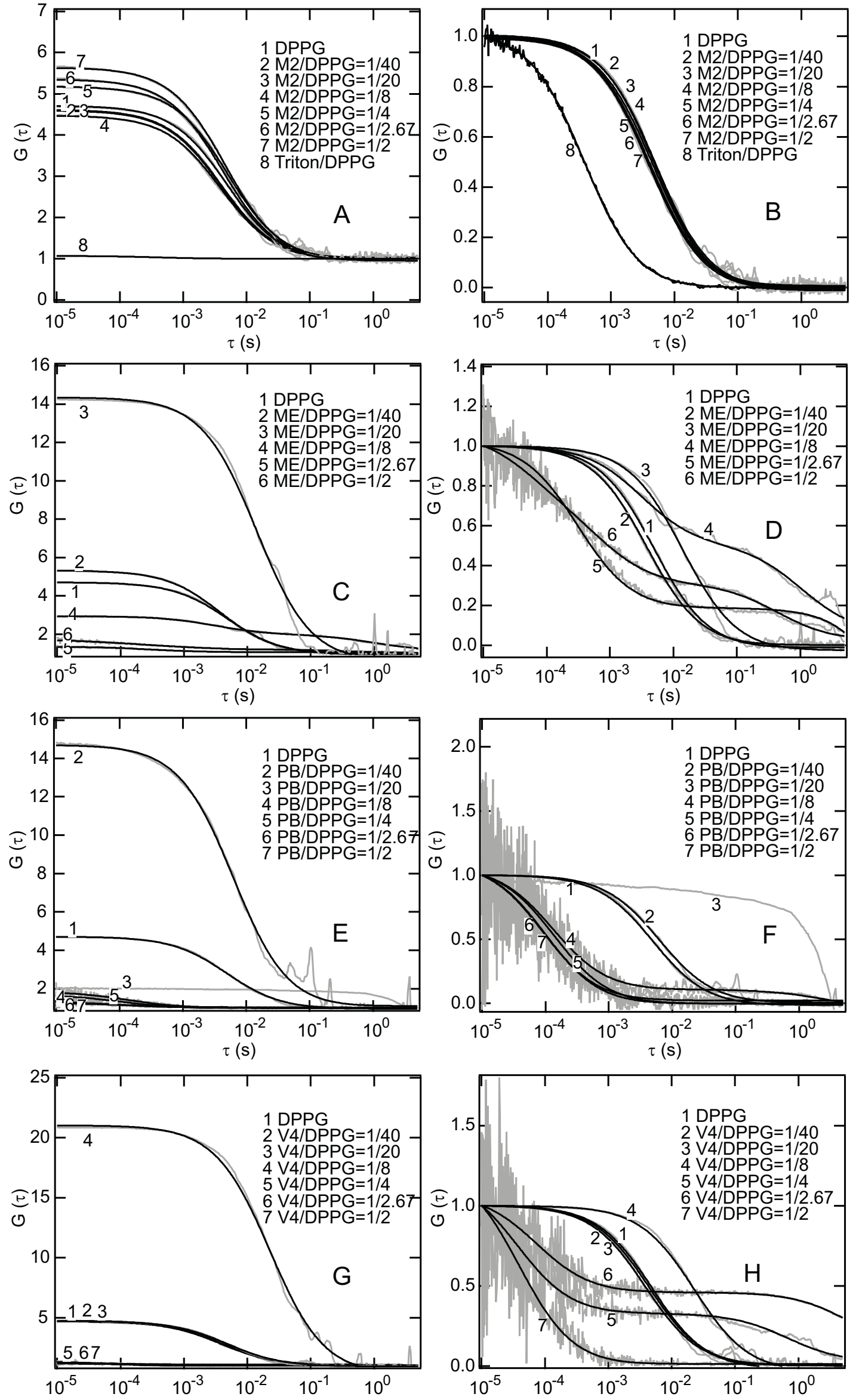

Fig. (S4). Interaction of four antimicrobial peptides with DPPG RLVs. The graphs on the left $(\mathbf{A}, \mathbf{C}, \mathbf{E}$ and $\mathbf{G})$ are the measured autocorrelation functions and their fits. The graphs on the right, $(\mathbf{B}, \mathbf{D}, \mathbf{F}$ and $\mathbf{H})$ depict normalized autocorrelation functions. 


\section{ABBREVIATIONS}

\begin{tabular}{|c|c|c|}
\hline $\mathrm{ACF}$ & $=$ & Autocorrelation function \\
\hline DPPG & $=$ & $\begin{array}{l}\text { 1,2-Dipalmitoyl-sn-Glycero-3-[Phospho-rac- } \\
\text { (1-glycerol)] }\end{array}$ \\
\hline FCS & $=$ & Fluorescence correlation spectroscopy \\
\hline LPS & $=$ & Lipopolysaccharides \\
\hline LUVs & $=$ & Large unilamellar vesicles \\
\hline M2 & $=$ & Magainin 2 \\
\hline ME & $=$ & Melittin \\
\hline $\mathrm{PB}$ & $=$ & Polymyxin B \\
\hline PBS & $=$ & Phosphate buffered saline \\
\hline $\mathrm{P} / \mathrm{L}$ & $=$ & Peptide/lipid ratio \\
\hline POPC & $=$ & $\begin{array}{l}\text { 1-Palmitoyl-2-Oleoyl-sn-Glycero-3- } \\
\text { Phosphocholine }\end{array}$ \\
\hline POPG & $=$ & $\begin{array}{l}\text { 1-Palmitoyl-2-Oleoyl-sn-Glycero-3- } \\
\text { [Phospho-rac-(1-glycerol)] }\end{array}$ \\
\hline REVs & $=$ & Rhodamine $6 \mathrm{G}$ entrapping LUVs \\
\hline Rho 6G & $=$ & Rhodamine $6 \mathrm{G}$ \\
\hline Rho-PE & $=$ & $\begin{array}{l}\text { 1,2-Dipalmitoyl-sn-Glycero-3-Phospho- } \\
\text { ethanolamine-N-(Lissamine Rhodamine B } \\
\text { Sulfonyl) (Ammonium Salt) }\end{array}$ \\
\hline $\mathrm{Vs}$ & $=$ & Rho-PE labeled LUVs \\
\hline V4 & $=$ & Cyclized CVKVQVKVGSGVKVQVKVC \\
\hline
\end{tabular}

\section{REFERENCES}

[1] Zasloff M. Antimicrobial peptides of multicellular organisms, Nature 2002; 415: 389-95

[2] Shai Y. Mechanism of the binding, insertion and destabilization of phospholipid bilayer membranes by $\alpha$-helical antimicrobial and cell non-selective membrane-lytic peptides. Biochim Biophys Acta 1999; 1462: 55-70.

[3] Mookherjee N, Hancock RE. Cationic host defence peptides: innate immune regulatory peptides as a novel approach for treating infections. Cell Mol Life Sci 2007; 64: 922-33.

[4] Brown KL, Hancock RE. Cationic host defense (antimicrobial) peptides. Curr Opin in Immunol 2006; 18: 24-30.

[5] Ganz T. Defensins: antimicrobial peptides of innate immunity. Nat Rev Immunol 2003; 3: 710-20.

[6] Hancock RE. Peptide antibiotics. Lancet 1997; 349: 418-22.

[7] Oren Z, Shai Y. Mode of action of linear amphipathic $\alpha$-helical antimicrobial peptides. Biopolymers 1998; 47: 451-63.

[8] Berkowitz BA, Bevins CL, Zasloff MA. Magainins: A new family of membrane-active host defense peptides. Biochem Pharmacol 1990; 39: 625-29.

[9] Zasloff M. Magainins, a class of antimicrobial peptides from Xenopus skin: isolation, characterization of two active forms, and partial cDNA sequence of a precursor. Proc Nat Acad Sci USA 1987; 84: 5449-53.

[10] Blondelle SE, Lohner K, Aguilar MI. Lipid-induced conformation and lipid-binding properties of cytolytic and antimicrobial peptides: determination and biological specificity. Biochim Biophys Acta 1999; 1462: 89-108.

[11] Ludtke SJ, He K, Wu Y, Huang HW. Cooperative membrane insertion of magainin correlated with its cytolytic activity. Biochim Biophys Acta 1994; 1190: 181-4.

[12] Matsuzaki K. Magainins as paradigm for the mode of action of pore forming polypeptides. Biochim Biophys Acta 1998; 1376: 391-400.

[13] Benachir T, Lafleur M. Study of vesicle leakage induced by melittin. Biochim Biophys Acta 1995; 1235: 452-60.
[14] Vogel H, Jahnig F. The structure of melittin in membranes. Biophys J 1986; 50: 573-82.

[15] Habermann E. Bee and wasp venoms. Science 1972; 177: 314-22.

[16] Thomas CJ, Surolia A. Kinetics of the interaction of endotoxin with polymyxin $\mathrm{B}$ and its analogs: a surface plasmon resonance analysis. FEBS Lett 1999; 445: 420-4.

[17] Morrison DC, Jacobs DM. Binding of polymyxin B to the lipid A portion of bacterial lipopolysaccharides. Immunochemistry 1976; 13: 813-18.

[18] Arnold TM, Forrest GN, Messmer KJ. Polymyxin antibiotics for gram-negative infections. Am J Health Syst Pharm 2007; 64: 81926.

[19] Thomas CJ, Surolia N, Surolia A. Surface plasmon resonance studies resolve the enigmatic endotoxin neutralizing activity of polymyxin B. J Biol Chem 1999; 274: 29624-7.

[20] Roes S, Seydel U, Gutsmann T. Probing the properties of lipopolysaccharide monolayers and their interaction with the antimicrobial peptide polymyxin B by atomic force microscopy. Langmuir 2005 ; 21: 6970-8.

[21] Oh D, Shin SY, Lee S, et al. Role of the hinge region and the tryptophan residue in the synthetic antimicrobial peptides, cecropin $\mathrm{A}(1-8)$-magainin 2(1-12) and its analogues, on their antibiotic activities and structures. Biochemistry 2000; 39: 11855-64.

[22] Javadpour MM, Juban MM, Lo WCJ, et al. De novo antimicrobial peptides with low mammalian cell toxicity. J Med Chem 1996; 39: 3107-13.

[23] Frecer V, Ho B, Ding JL. De novo design of potent antimicrobial peptides. Antimicrob Agents Chemother 2004; 48: 3349-57.

[24] Yu L, Ding JL, Ho B, Wohland T. Investigation of a novel artificial antimicrobial peptide by fluorescence correlation spectroscopy: An amphipathiccationic pattern is sufficient for selective binding to bacterial type membranes and antimicrobial activity. Biochim Biophys Acta 2005; 1716: 29-39.

[25] Falla TJ, Karunaratne DN, Hancock RE. Mode of action of the antimicrobial peptide indolicidin. J Biol Chem 1996; 271: 19298303.

[26] Piers KL, Brown MH, Hancock RE. Improvement of outer membrane-permeabilizing and lipopolysaccharide-binding activities of an antimicrobial cationic peptide by C-terminal modification. Antimicrob Agents Chemother 1994; 38: 2311-6.

[27] Yang L, Harroun TA, Weiss TM, Ding L, Huang HW. Barrel-stave model or toroidal model? A case study on melittin pores. Biophys J 2001; 81: 1475-85.

[28] Ludtke SJ, He K, Heller WT, Harroun TA, Yang L, Huang HW. Membrane pores induced by magainin. Biochemistry 1996; 35: 13723-8.

[29] Pramanik A, Thyberg P, Rigler R. Molecular interactions of peptides with phospholipid vesicle membranes as studied by fluorescence correlation spectroscopy. Chem Phys Lipids 2000; 104: $35-$ 47.

[30] McIntyre JC, Sleight RG. Fluorescence assay for phospholipid membrane asymmetry. Biochemistry 1991; 30: 11819-27.

[31] Rusu L, Gambhir A, McLaughlin S, Radler J. Fluorescence correlation spectroscopy studies of peptide and protein binding to phospholipid vesicles. Biophys J 2004; 87: 1044-53.

[32] Miszta A, Machan R, Benda A, Ouellette AJ, Hermens WT, Hof M. Combination of ellipsometry, laser scanning microscopy and Zscan fluorescence correlation spectroscopy elucidating interaction of cryptdin-4 with supported phospholipid bilayers. J Pept Sci 2007

[33] Magde D, Elson E, Webb WW. Thermodynamic fluctuations in a reacting system-measurement by fluorescence correlation spectroscopy. Phys Rev Lett 1972; 29: 705-8.

[34] Maiti S, Haupts U, Webb WW. Fluorescence correlation spectroscopy: Diagnostics for sparse molecules. Proc Nat Acad Sci USA 1997; 94: 11753-7.

[35] Krichevsky O, Bonnet G. Fluorescence correlation spectroscopy: the technique and its applications. Rep Prog Phys 2002; 65: 251-97.

[36] Hess ST, Huang S, Heikal AA, Webb WW. Biological and chemical applications of fluorescence correlation spectroscopy: A review. Biochemistry 2002; 41: 697-705.

[37] Widengren J, Rigler R. Fluorescence correlation spectroscopy as a tool to investigate chemical reactions in solutions and on cell surfaces. Cell Mol Biol 1998; 44: 857-79. 
[38] Gosch M, Rigler R. Fluorescence correlation spectroscopy of molecular motions and kinetics. Adv Drug Delivery Rev 2005; 57: 169-90.

[39] Berland KM. Fluorescence correlation spectroscopy: a new tool for quantification of molecular interactions. Methods Mol Biol 2004; 261: 383-98

[40] Haustein E, Schwille P. Single-molecule spectroscopic methods. Curr Opin Struct Biol 2004; 14: 531-40.

[41] Kim SA, Schwille P. Intracellular applications of fluorescence correlation spectroscopy: prospects for neuroscience. Curr Opin Neurobiol 2003; 13: 583-90.

[42] Haustein E, Schwille P. Ultrasensitive investigations of biological systems by fluorescence correlation spectroscopy. Methods 2003; 29: 153-66.

[43] Ehrenberg M, Rigler R. Rotational brownian motion and fluorescence intensify fluctuations. Chem Phys 1974; 4: 390-401.

[44] Kask P, Günther R, Axhausen P. Statistical accuracy in fluorescence fluctuation experiments. Eur Biophys J 1997; 25: 163-9.

[45] Wohland T, Rigler R, Vogel H. The standard deviation in fluorescence correlation spectroscopy. Biophys J 2001; 80: 2987-99.

[46] Aragon SR, Pecora R. Fluorescence correlation spectroscopy as a probe of molecular dynamics. J Chem Phys 1976; 64: 1791-803.

[47] Meseth U, Wohland T, Rigler R, Vogel H. Resolution of fluorescence correlation measurements. Biophys J 1999; 76:1619-31.

[48] Yu L, Tan M, Ho B, Ding JL, Wohland T. Determination of critical micelle concentrations and aggregation numbers by fluorescence correlation spectroscopy: Aggregation of a lipopolysaccharide. Anal Chim Acta 2006; 556: 216-25.

[49] Thompson NL. Topics in fluorescence spectroscopy. Plenum Press, New York, 1991.
[50] Magzoub M, Oglecka K, Pramanik A, Eriksson LEG, Graslund A. Membrane perturbation effects of peptides derived from the $\mathrm{N}$ termini of unprocessed prion proteins. Biochim Biophys Acta 2005; 1716: 126-36.

[51] Matsuzaki K, Murase O, Fujii N, Miyajima K. An Antimicrobial peptide, magainin 2, induced rapid flip-flop of phospholipids coupled with pore formation and peptide translocation. Biochemistry 1996; 35: 11361-8

[52] Matsuzaki K, Yoneyama S, Miyajima K. Pore formation and translocation of melittin. Biophys J 1997; 73: 831-8.

[53] Tossi A, Sandri L, Giangaspero A. Amphipathic, $\alpha$-helical antimicrobial peptides. Biopolymers 2000; 55: 4-30.

[54] Rana FR, Blazyk J. Interactions between the antimicrobial peptide, magainin 2, and Salmonella typhimurium lipopolysaccharides. FEBS Lett 1991; 293: 11-5.

[55] Wieprecht T, Apostolov O, Seelig J. Binding of the antibacterial peptide magainin 2 amide to small and large unilamellar vesicles. Biophys Chem 2000; 85: 187-98.

[56] Rana FR, Macias EA, Sultany CM, Modzrakowski MC, Blazyk J. Interactions between magainin 2 and Salmonella typhimurium outer membranes: effect of lipopolysaccharide structure. Biochemistry $1991 ; 30: 5858-66$

[57] Wenk MR, Seelig J. Magainin 2 amide interaction with lipid membranes: Calorimetric detection of peptide binding and pore formation. Biochemistry 1998; 37: 3909-16.

[58] Rex S, Schwarz G. Quantitative studies on the melittin-induced leakage mechanism of lipid vesicles. Biochemistry 1998; 37: 233645 .

(C) Yu et al.; Licensee Bentham Open.

This is an open access article distributed under the terms of the Creative Commons Attribution License (http:/creativecommons.org/licenses/by/2.5/), which permits unrestrictive use, distribution, and reproduction in any medium, provided the original work is properly cited. 